\title{
Polynomial monads and delooping of mapping spaces
}

\author{
M. A. Batanin* \\ F. De Leger ${ }^{\dagger}$ \\ michael.batanin@mq.edu.au florian.deleger@mq.edu.au
}

January 16, 2020

\begin{abstract}
We extend some classical results - such as Quillen's Theorem A, the Grothendieck construction, Thomason's theorem and the characterisation of homotopically cofinal functors - from the homotopy theory of small categories to polynomial monads and their algebras.

As an application we give a categorical proof of the Dwyer-Hess and Turchin results concerning the explicit double delooping of spaces of long knots.
\end{abstract}

\section{Contents}

\section{Polynomial monads and homotopy theory 2}

1 Introduction $\quad[2$

2 Polynomial monads $\quad 7$

3 Internal algebras and Grothendieck construction 10

4 Classifiers for maps between polynomial monads $\quad 14$

5 Homotopy theory of algebras and classifiers $\quad 17$

6 Twisted Boadrman-Vogt tensor product
and Thomason's theorem

7 Homotopy pushouts of classifiers

8 Mapping spaces between pointed algebras of polynomial monads 28

*Macquarie University, North Ryde, 2109 Sydney, Australia

${ }^{\dagger}$ Macquarie University, North Ryde, 2109 Sydney, Australia

2010 Mathematics Subject Classification. 18D20 , 18D50, 55P48 
II Applications 31

9 Multiplicative operads, bimodules and weak bimodules 31

10 First cofinality theorem

11 Second cofinality theorem 46

12 Dwyer-Hess-Turchin's delooping theorems 5

Part I

\section{Polynomial monads and homotopy theory}

\section{Introduction}

The homotopy theory of small categories is a product of decades of development by many prominent mathematicians; to name a few: Quillen, Grothendieck and Thomason 14, 19, 22. More recent significant progress is the work of Maltsiniotis and Cisinski [17, 8]. The theory provides a vital formalism for many applications in algebraic geometry, algebraic $K$-theory and algebraic topology.

In this paper we show that some of the fundamental constructions and results from the homotopy theory of small categories are still valid in the larger context of the category of finitary polynomial monads. The theory of finitary polynomial monads (equivalently known as $\Sigma$-free coloured Set-operads) is a multivariable extension of the theory of small categories. Indeed, a small category $\mathrm{C}$ with the set of objects $I$ determines an endofunctor $C: \operatorname{Set}^{I} \rightarrow \operatorname{Set}^{I}$, where $\operatorname{Set}^{I}$ is the category of $I$-indexed collections of sets:

$$
C(X)(i)=\coprod_{j} \mathrm{C}(j, i) \times X(j)
$$

where $\mathrm{C}(j, i)$ is the set of morphisms in $\mathrm{C}$ from $j$ to $i$. It is easy to see that the functor $C$ preserves connected limits. The category structure of $\mathrm{C}$ amounts then to a structure of cartesian monad on the functor $C$. The last conditions mean that the unit and multiplication of this monad are cartesian natural transformations; that is, all naturality squares are pullbacks. The category of algebras for this monad is isomorphic to the category of covariant presheaves Set $^{\mathrm{C}}$.

Finitary polynomial monads can be defined as cartesian monads whose underlying functor is a coproduct of sets which involve multivariable summands like $B\left(j_{1}, \ldots, j_{k} ; i\right) \times X\left(j_{1}\right) \times \ldots \times X\left(j_{k}\right)$ with finitely many factors. The category of algebras of such a monad is the category of $I$-collections equipped with the operations

$$
B\left(j_{1}, \ldots, j_{k} ; i\right) \times X\left(j_{1}\right) \times \ldots \times X\left(j_{k}\right) \rightarrow X(i)
$$


which satisfy appropriate associativity and unitarity conditions. One can also give the structure of an algebra by specifying a family of maps

$$
b: X\left(j_{1}\right) \times \ldots \times X\left(j_{k}\right) \rightarrow X(i), b \in B\left(j_{1}, \ldots, j_{k} ; i\right) .
$$

An important fact is: the category of algebras of a finitary polynomial monad can be defined in any symmetric monoidal category $\mathcal{E}$. Indeed, it suffices to replace the cartesian product of sets in the definition of algebra by the tensor product of objects in $\mathcal{E}$.

It was observed by the first author in 3 , that the algebras of a polynomial monad in the symmetric monoidal category $(\mathrm{Cat}, \times, 1)$ of small categories (called categorical algebras) play a special role. For such algebras the theory can be internalised; that is, one can consider a kind of algebra (called internal algebra) inside a categorical algebra of a polynomial monad. Formally, an internal $T$ algebra inside a categorical $T$-algebra $A$ is defined as a lax $T$-algebra map from the terminal $T$-algebra 1 to $A$.

A good example to have in mind is the category of monoids in any monoidal category. We consider a monoidal category as a categorical (pseudo)algebra of a finitary polynomial monad (the free monoid monad) $M$ given by the geometric series $M(X)=\amalg_{n>0} X^{n}$. It is well known then that a monoid in a monoidal category $A$ is the same thing as a lax-monoidal functor from the terminal monoidal category 1 to $A$; that is, a lax $M$-algebras map $1 \rightarrow A$.

Remark 1.1. More precisely, the categorical algebras of $M$ are strict monoidal categories. However, this difference between strict monoidal categories and general monoidal categories does not play much role in our theory due to Mac Lane's coherence theorem.

A classical observation of Lawvere is that the theory of monoids is represented by the monoidal category of finite ordinals $\Delta_{\text {alg }}$ in the sense that this monoidal category is freely generated by a monoid (the terminal ordinal) inside it. This means that a monoid in a monoidal category $A$ is the same as a strict monoidal functor from $\Delta_{\text {alg }}$ to $A$.

It was shown in $[3$ that this observation of Lawvere has a far reaching generalisation: for any cartesian map between cartesian monads $f: S \rightarrow T$, there exists a categorical $T$-algebra $T^{S}$ with a nice universal property: it is freely generated by an internal $S$-algebra. We called this algebra the classifier of internal $S$-algebras inside categorical T-algebras.

The theory of classifiers provides a link between Grothendieck's homotopy theory and polynomial monads. For example, if $f: S \rightarrow T$ is a functor between small categories (interpreted as a map between the corresponding cartesian monads) then $T^{S}$ is the covariant Cat-valued presheaf on $T$ which associates to an object $i \in T$ the comma (or slice) category $f / i$. This slice category construction is one of the main tools of the homotopy theory of small categories.

The theory of internal algebras classifiers for polynomial monads and its applications was developed further by the first author and Clemens Berger in [5. An application of this theory to the Baez and Dolan stabilization hypothesis for higher dimensional categories was found in [1]. It was observed that the homotopy 
type of the classifier for $f: S \rightarrow T$ can tell us a lot about the homotopy behaviour of the adjoint pair of functors between simplicial algebras of $T$ and $S$ induced by $f$, very much like in the homotopy theory of small categories where the homotopy type of slices of a functor provides important information about homotopy Kan extensions along this functor.

In this paper we take this analogy seriously and develop a formalism extending that of the homotopy theory of small categories. The main ingredients of our new formalism are :

1. An analogue of the Grothendieck construction for a polynomial monad and interpretation of the classifier construction as its left adjoint;

2. An analogue of Quillen's Theorem A for polynomial monads;

3. The analogue of the characterisation of homotopically cofinal functors in terms of preservation of homotopy limits.

4. A generalisation of Thomason's theorem about the homotopy colimit of nerves of a diagram of small categories.

It turns out that this extended formalism provides some extra flexibility which is not achievable in the classical setting of small categories. For example, one can add constants to the theory, which turns out to be very useful in the study of homotopy mapping spaces between simplicial algebras.

As an illustration of the power of this formalism we give a new proof of the Dwyer-Hess-Turchin result on explicit double delooping of the space of long knots [11, 23]. Our work was, in fact, inspired by the paper of Turchin [23].

The space $\overline{E m b}\left(\mathbb{R}^{1}, \mathbb{R}^{m}\right)$ of long knots modulo immersions is a homotopy fiber of the map

$$
\operatorname{Emb}\left(\mathbb{R}^{1}, \mathbb{R}^{m}\right) \rightarrow \operatorname{Imm}\left(\mathbb{R}^{1}, \mathbb{R}^{m}\right)
$$

where $\operatorname{Emb}(-,-)$ is the space of embeddings and $\operatorname{Imm}(-,-)$ is the space of immersions with compact support (that is, it is equal to the standard embedding outside of a compact subspace).

Dwyer-Hess and independently Turchin proved the following statement: for $m>3$, there is a weak equivalence of spaces

$$
\overline{E m b}\left(\mathbb{R}^{1}, \mathbb{R}^{m}\right) \sim \Omega^{2} M a p_{\mathrm{Op}}\left(\mathcal{D}_{1}, \mathcal{D}_{m}\right)
$$

where $\mathcal{D}_{k}$ is a $E_{k}$-operad (that is, any operad homotopy equivalent to the little $k$-disks operad), $\operatorname{Map}_{\mathrm{Op}}(-,-)$ is the homotopy mapping space in the category of symmetric operads and $\Omega$ is the loop space functor.

Both proofs use an earlier result of Sinha 20] about the weak equivalence:

$$
\overline{\operatorname{Emb}}\left(\mathbb{R}^{1}, \mathbb{R}^{m}\right) \sim \widetilde{\operatorname{Tot}}(\mathcal{K})
$$

where $\widetilde{\operatorname{Tot}}(\mathcal{K})$ is the cosimplicial totalization of the Kontsevich operad $\mathcal{K}$. This construction is possible because $\mathcal{K}$ is not only an $E_{m}$-operad but it is also multiplicative; that is, it is equipped with a map of non-symmetric operads $\mathcal{A} s s \rightarrow d_{1} \mathcal{K}$, 
where $\mathcal{A s s}$ is the terminal non-symmetric operad and $d_{1}(-)$ is the functor of dessymmetrisation (that is, the functor forgetting the symmetric groups actions).

In fact, Dwyer-Hess and Turchin established the following more general delooping result:

Theorem 1.2. For any multiplicative reduced non-symmetric operad $\mathcal{O}$, there are two weak equivalences of mapping spaces:

$$
\operatorname{Map}_{\text {Bimod }}(\mathcal{A} s s, \mathcal{O}) \sim \Omega M a p_{\mathrm{NOp}}(\mathcal{A} s s, \mathcal{O})
$$

and

$$
\operatorname{Map}_{\text {WBimod }}(\mathcal{A} s s, \mathcal{O}) \sim \Omega M a p_{\text {Bimod }}(\mathcal{A} s s, \mathcal{O}),
$$

where mapping spaces are taken in the model categories of non-symmetric operads NOp, Ass-bimodules Bimod and weak Ass-bimodules WBimod. Reducedness means that $\mathcal{O}_{0}$ and $\mathcal{O}_{1}$ are both contractible spaces.

This theorem is applicable to delooping the space of long knots because (as several people observed) the category WBimod is isomorphic to the category of cosimplicial objects and, hence, the (homotopy) cosimplicial totalization functor is the homotopy mapping space from the terminal weak bimodule.

The proofs of this theorem by Dwyer-Hess and Turchin are both based on homotopy theory but of different flavours. Turchin uses some very explicit cofibrant resolutions for operads, bimodules and weak bimodules and then constructs all necessary higher homotopies by hand. Dwyer and Hess use model theoretical argument related to moduli spaces (in fact they prove a more general statement about relations between homotopy mapping spaces of monoids and bimodules). Unfortunately, both proofs are very technical and do not provide a clear conceptual explanation of the result. Consequently both proofs are hard to generalise to other situations where we want to study the delooping of mapping spaces; for example, for delooping the higher dimensional spaces of embeddings.

We approach this question by observing that all three categories NOp, Bimod and WBimod are categories of algebras of appropriate polynomial monads. The mapping spaces like $M a p_{\mathrm{NOp}}(\mathcal{A s s}, \mathcal{O})$ are then the 'derived versions' of the category of internal algebras. So the statement of the theorem can be conceptually understood in the setting of internal algebras.

For example, the first delooping statement can be understood at the outset through the following 'baby' case. Suppose we are given two maps of nonsymmetric operads $\mathcal{A} s s \rightarrow \mathcal{O}$ in Cat. One can then construct an $\mathcal{A} s s$-bimodule out of $\mathcal{O}$ using the first map to define a left action of $\mathcal{A} s s$ and the second map to define a right action. Now, suppose that $\mathcal{O}$ is a groupoid in each degree and $\mathcal{O}_{1}$ is a contractible groupoid. Then one can prove by hand that the process above has an inverse; that is, any bimodule structure on the collection of categories $\mathcal{O}$ is obtained from two maps of operads $\mathcal{A} s s \rightarrow \mathcal{O}$. In general, Turchin-Dwyer-Hess delooping is essentially the above statement where $\mathcal{O}$ is now an operad in $\omega$-groupoids. Of course, in this case the inverse functor reconstructs two operadic maps as well as an operadic structure on $\mathcal{O}$, but only up to higher homotopies. In our formalism 
this statement is equivalent to the statement that the map of polynomial monads

$$
\mathrm{Bimod}_{+} \rightarrow \mathrm{NOp}_{* *}
$$

is homotopically cofinal (Theorem 10.2). Here, Bimod ${ }_{+}$is the polynomial monad for $\mathcal{A} s s$-bimodules with an additional distinguished point in the degree 1 component and $N O p_{* *}$ is the polynomial monad for double multiplicative operads; that is, non-symmetric operads equipped with two maps from $\mathcal{A} s s$.

We believe that the possibility of using the above kind of reasoning to conceptually understand a problem, and then apply formal high level homotopy theory language to finish the proof, illustrates an important powerful feature of our approach. We expect the technique to be very useful in future applications.

Here is the plan of the paper. Part 1 is devoted to the formalism of the homotopy theory of polynomial monads. We define the category of Internal algebras of polynomial monads in Section 3. We then show that there is an analogue of the Grothendieck construction for polynomial monads which produce a polynomial monad map out of a categorical algebra of a polynomial monad. We then interpret the category of internal algebras as a category of sections of Grothendieck constructions. In this sense the category of internal algebras can be thought as the nonabelian cohomology of the polynomial monad. The category of relative internal algebras also admits an interpretations in terms of a category of liftings. In Section 3 we recall the definition of internal algebra classifiers and their construction in terms of a codescent object. Our new result here is that the classifier construction is the left 2-adjoint to the Grothendieck construction.

In Section 5 we relate internal algebra classifiers with the homotopy theory of simplicial algebras over a polynomial monad. Here we prove Quillen's Theorem A for polynomial monads. We also introduce the notion of homotopically cofinal maps between polynomial monads and show that these maps can be characterised in terms of maps between mapping spaces between algebras very much as homotopically cofinal functor can be characterised as functors restriction along which preserves homotopy colimits.

In Section 6 we develop yet another version of Grothendieck construction which we call twisted Boardman-Vogt tensor product. It is interesting that these two versions of Grothendieck constructions coincide in the 2-category of small categories. We prove then a generalisation to polynomial monads of Thomason's theorem about the colimit of the diagram of nerves of small categories. In Section 7 we apply this criteria to introduce a notion of homotopically cofinal square of polynomial monads. These are exactly the commutative squares of polynomial monads which induce the homotopy pushouts of nerves of classifiers over any fixed polynomial monad.

In Section 8 we prove some useful results about formal delooping of homotopy mapping spaces between pointed algebras of polynomial monads. These results will play main role in our approach to the proof of the Dwyer-Hess-Turchin theorems in Part 2 of our paper. We must add that most of our results about homotopy mapping spaces can be proved for (semi) model categories of algebras of polynomial monads in a monoidal model category satisfying some very moderate assumptions. 
We do not do it in this paper just because it would make the proofs more technical and longer at the expense of clarity of exposition of the main new ideas.

In Section 9 we briefly remind the reader about multiplicative operads, bimodules and weak bimodules, and show that there are polynomial monads for all these categories.

In Section 10 we prove a result about homotopy cofinality of a certain map between polynomial monads (Theorem 10.2). This result is a vast generalisation of the Dwyer-Hess-Turchin result where we compare mapping spaces between cospans of operads and bimodules over different operads. This is exactly this theorem which provides a conceptual explanation of the existence of Dwyer-Hess and Turchin delooping. An analogous result holds for mapping spaces between cospans of bimodules and two sided weak bimodules.

In Section 11 we show how cofinality of the maps $\operatorname{Bimod}_{+} \rightarrow N O p_{* *}$ and WBimod $_{+} \rightarrow$ Bimod $_{* *}$ follows from Theorem 10.2. Finally, in Section 12 we provide a proof of the Dwyer-Hess-Turchin theorem which is now a relatively simple consequence of our formal delooping theorems and the second cofinality Theorem 11.1

Latest development. A significant progress in explicit delooping of the embedding spaces was made recently in [7, 9, 10. The approach of Boavida and Weiss [7] is more topological, whereas our approach is categorical and combinatorial and is closer to the Dwyer-Hess and Ducoulombier-Turchin approaches in 9, 10. As it is noticed in [7] : ' . . . the Dwyer-Hess result is a theorem about fairly general operads and as such it has a different scope and applicability from our result'. There are, however, very interesting connections between all these approaches and we are going to address it in a future paper.

\section{Polynomial monads}

Definition $2.1([16,13,5)$. A finitary polynomial $P$ is a diagram in Set of the form

$$
J \longleftarrow \stackrel{s}{\longleftrightarrow} B \stackrel{p}{\longrightarrow} I
$$

where $p^{-1}(b)$ is a finite set for any $b \in B$.

Each polynomial $P$ generates a functor called polynomial functor between functor categories

$$
\underline{P}: \operatorname{Set}^{J} \rightarrow \operatorname{Set}^{I}
$$

which is defined as the composite functor

$$
\operatorname{Set}^{J} \longrightarrow s^{*} \longrightarrow \operatorname{Set}^{E} \longrightarrow p_{*} \longrightarrow \operatorname{Set}^{B} \longrightarrow t_{!} \operatorname{Set}^{I}
$$

where we consider the sets $J, E, B, I$ as discrete categories and $s^{*}$ is the restriction functor and $p_{*}$ and $t_{\text {! }}$ are the right and left Kan extensions correspondingly. Explicitly the functor $\underline{P}$ is given by the formula

$$
\underline{P}(X)_{i}=\coprod_{b \in t^{-1}(i)} \prod_{e \in p^{-1}(b)} X_{s(e)},
$$


which explains the name 'polynomial': its values are sums of products of formal variables.

A cartesian morphism between polynomial functors is a natural transformation between the functors such that each naturality square is a pullback. One can prove that such a cartesian morphism is determined by a commutative diagram in Set

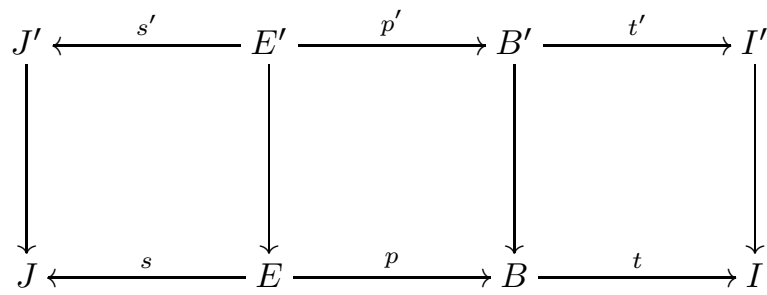

such that the middle square is a pullback.

Composition of finitary polynomial functors is again a finitary polynomial functor. Sets, finitary polynomial functors and their cartesian morphisms form a 2category Poly.

Definition 2.2. A finitary polynomial monad is a monad in the 2-category Poly.

Remark 2.3. Finitary polynomial functors preserve filtered colimits and pullbacks. Polynomial monads are cartesian; that is, their underlying functors preserve pullbacks and their units and multiplications are cartesian natural transformations.

Remark 2.4. One can consider more general polynomial functors of non-finitary type. Since in this paper we shall not need these more general functors, we use the term polynomial monad for finitary polynomial monad.

For a polynomial monad $T$

$$
I \longleftrightarrow \text { s } E \longrightarrow B \longrightarrow
$$

we will call the set $I$ the set of colours of $T$, the set $B$ the set of operations of $T$, the set $E$ the set of marked operations of $T$, the map $t$ the target map and the map $s$ the source map. The map $p$ will be called the middle map of $T$.

Explicitly, the structure of polynomial monad is given by a family of elements (units) $1_{i} \in B$ for all $i \in I$ such that $t\left(1_{i}\right)=i, s\left(p^{-1}\left(1_{i}\right)\right)=\{i\}$, and a composite $\mu_{T}\left(b ; b_{1}, \ldots, b_{k}\right)$ for each $b \in B$, and each list of elements $b_{1}, \ldots b_{k} \in B$ together with a bijection $\eta:\{1, \ldots, k\} \rightarrow s\left(p^{-1}(b)\right)$ such that $t\left(b_{m}\right)=\eta(m)$. These data should satisfy unitarity, associativity and equivariancy conditions. Polynomial monads and their cartesian maps form a category PMon.

Example 2.5. One can consider a small category $C$ with the set of objects $I$ and set of morphisms $B$ as a polynomial monad

$$
I \stackrel{s}{\longleftrightarrow} B \stackrel{i d}{\longrightarrow} B \stackrel{t}{\longrightarrow} I
$$

where $s$ and $t$ are the usual source and target maps. This gives us a full embedding of categories Cat $\rightarrow$ PMon. This embedding has a right adjoint which for a polynomial monad $T$ returns its submonad of unary operations. 
Example 2.6 ([5]). The free monoid monad is a polynomial monad represented by the diagram

$$
1 \longleftrightarrow{ }^{2}-r^{*} \longrightarrow \operatorname{ptr} \longrightarrow
$$

where $L t r$ is the set of isomorphism classes of linear trees (or equally the set of all ordinals $\{0<1<\ldots<n\})$, the set $L t r^{*}$ is the set of linear trees with one vertex marked (equivalently the set of all ordinals $\left\{0<1<\ldots<k^{*}<\ldots<n\right\}$ ), the set of colours is the one object set. The middle map forgets the marking. The multiplication in the monad is generated by insertion of a linear tree to the marked vertex of another tree.

Example 2.7. Recall that a non-symmetric operad $\mathcal{O}$ in a symmetric monoidal category $(\mathcal{E}, \otimes, e)$ is given by

- an object $\mathcal{O}_{n}$ in $\mathcal{E}$ for all integers $n \geq 0$

- a morphism $\epsilon: e \rightarrow \mathcal{O}_{1}$ called unit

- morphisms

$$
m: \mathcal{O}_{k} \otimes \mathcal{O}_{n_{1}} \otimes \ldots \otimes \mathcal{O}_{n_{k}} \rightarrow \mathcal{O}_{n_{1}+\ldots+n_{k}}
$$

called multiplication

such that the usual associativity and unitarity conditions are satisfied.

The polynomial monad $N O p$ for non-symmetric operads was described in 5 , [16. The corresponding polynomial is :

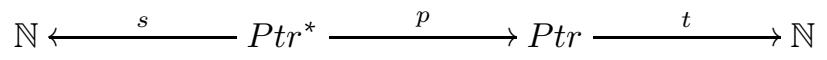

Here, $P t r, P t r^{*}$ are the sets of isomorphism classes of planar trees, and planar trees with a marked vertex respectively. The middle map forgets the marked point, the source map is given by the number of incoming edges for the marked point and the target map associates to a tree its number of leaves. The multiplication in this monad is generated by insertion of a tree inside a marked point.

Let $\mathcal{E}$ be a cocomplete symmetric monoidal category and $T$ be a polynomial functor. One can construct a functor $\underline{T}^{\mathcal{E}}: \mathcal{E}^{I} \rightarrow \mathcal{E}^{I}$ given by a formula similar to (3):

$$
\underline{T}^{\mathcal{E}}(X)_{i}=\coprod_{b \in t^{-1}(i)} \bigotimes_{e \in p^{-1}(b)} X_{s(e)} .
$$

If $I=J$ and $T$ was given the structure of a polynomial monad then $\underline{T}^{\mathcal{E}}$ would acquire a structure of monad on $\mathcal{E}^{I}$. This last category will be called the category of $I$-collections in $\mathcal{E}$. The category of Set-collections often will be called simply the category of $I$-collections and the category of Cat-collection will be called the category of categorical $I$-collections.

Definition 2.8. The category of algebras of a polynomial monad $T$ in a cocomplete symmetric monoidal category $\mathcal{E}$ is the category of algebras of the monad $\underline{T}^{\mathcal{E}}$. 
Explicitly, an $\mathcal{E}$-algebra $A$ of a polynomial monad $T$ is given by a collection $A_{i} \in \mathcal{E}, i \in I$, equipped with the following structure maps:

$$
m_{(b, \sigma)}: A_{s(\sigma(1))} \otimes \ldots \otimes A_{s(\sigma(k))} \rightarrow A_{t(b)}
$$

for each $b \in B$, and each bijection $\sigma:\{1, \ldots, k\} \rightarrow p^{-1}(b)$ which satisfy some appropriate associativity, unitarity and the following equivariancy condition [5. If $\sigma^{\prime}:\{1, \ldots, k\} \rightarrow p^{-1}(b)$ is a bijection then the following triangle commutes:

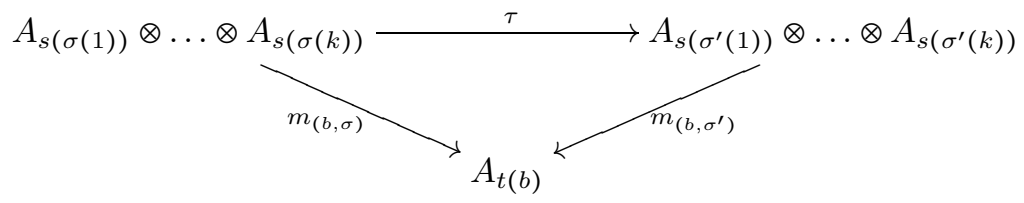

where $\tau$ is the action of the permutation $\left(\sigma^{\prime}\right)^{-1} \circ \sigma$.

Remark 2.9. The presence of the linear ordering of $p^{-1}(b)$ in the formula above is necessary to fix an order of tensor products. The equivariancy condition assures that the structure maps do not depend on the ordering [5]. This is closely related to the fact that the category of polynomial monads is equivalent to the category of $\Sigma$-free symmetric operads. The category of algebras of a polynomial monad is isomorphic to the category of algebras of the corresponding $\Sigma$-free operad [5].

\section{Internal algebras and Grothendieck construc- tion}

Algebras of a polynomial monad $T$ in the symmetric monoidal category of small categories $($ Cat, $\times, 1)$ will play a special role. We will call them categorical algebras of $T$. The category of categorical algebras of $T$ is isomorphic to the category of internal categories in the category of $T$-algebras of $T$ (in Set). The category of categorical $T$-algebras is naturally a 2 -category. We will use this fact but preserve the notation $\operatorname{Alg}_{T}$ (Cat) for this 2-category.

A terminal internal category has a unique $T$-algebra structure for any polynomial monad $T$; the latter promotes it to a terminal categorical $T$-algebra. From now on all terminal objects will be denoted 1 hoping that this will cause no confusion.

The following definitions are taken from [3] and [5].

Definition 3.1. Let $A$ be a categorical T-algebra for a polynomial monad $T$.

$A n$ internal $T$-algebra in $A$ is a lax morphism of categorical $T$-algebras from the terminal categorical $T$-algebra to A.

Internal T-algebras in $A$ and T-natural transformations form a category $\operatorname{Int}_{T}(A)$ and this construction extends to a 2-functor $\operatorname{Int}_{T}: \mathrm{Alg}_{T}$ (Cat) $\rightarrow$ Cat.

An internal $T$-algebra in a categorical $T$-algebra $A$ can be explicitly given by a collection of objects $a_{i} \in A_{i}$ together with a morphism

$$
\mu_{(b, \sigma)}: m_{(b, \sigma)}\left(a_{s(\sigma(1))}, \ldots, a_{s(\sigma(k))}\right) \rightarrow a_{t(b)},
$$


for each operation $(b, \sigma)$, which satisfies obvious associativity, unitarity and equivariancy conditions. Here, $m_{(b, \sigma)}$ is the structure functor of $A$.

Given a cartesian map of polynomial monads $f: S \rightarrow T$ we have a restriction 2 -functor $f^{*}: \operatorname{Alg}_{T}($ Cat $) \rightarrow \operatorname{Alg}_{S}($ Cat $)$.

Definition 3.2. Let $A$ be a categorical T-algebra for a polynomial monad $T$.

$A n$ internal $S$-algebra in $A$ is a lax morphism of categorical $S$-algebras from the terminal categorical $S$-algebra to $f^{*}(A)$.

Internal $S$-algebras in $A$ and $S$-natural transformations form a category $\operatorname{Int}_{S}(A)$ and this construction extends to a 2-functor

$$
\operatorname{Int}_{S}: \operatorname{Alg}_{T}(\text { Cat }) \rightarrow \text { Cat. }
$$

We will also need the following generalisation of the classical Grothendieck construction for categories to polynomial monads. Let $T$ be a polynomial monad and let $A$ be a categorical algebra for it. We construct a new polynomial monad $\int A$ as follows. The set of colours of $\int A$ is the set of pairs $(i, a)$ where $a$ is an object of the category $A_{i}$. An operation consists of:

1. An element $b \in B$;

2. For each element $e \in p^{-1}(b)$ an object $a_{e} \in A_{s(e)}$;

3. An object $y \in A_{t(b)}$;

4. A morphism $f_{(b, \sigma)}: m_{(b, \sigma)}\left(a_{\sigma(1)}, \ldots, a_{\sigma(k)}\right) \rightarrow y$ in $A_{t(b)}$ for each $\sigma:\{1, \ldots, k\} \rightarrow$ $p^{-1}(b)$, which satisfies the following equivariancy condition. If $\sigma^{\prime}:\{1, \ldots, k\} \rightarrow$ $p^{-1}(b)$, and $\tau\left(a_{\sigma(1)}, \ldots, a_{\sigma(k)}\right)=\left(a_{\sigma^{\prime}(1)}, \ldots, a_{\sigma^{\prime}(k)}\right)$ then

$$
f_{(b, \sigma)}=f_{\left(b, \sigma^{\prime}\right)} .
$$

Obviously, to specify an operation it suffices to know the morphism $f_{(b, \sigma)}$. A marked operation in $\int A$ is an operation in which one of the elements $e \in p^{-1}(b)$ is marked. As usual, the middle map forgets about marking. The target map of the monad $\int A$ is the pair $(t(b), y)$ and the source map is $\left(s(e), a_{e}\right)$ where $e$ is the marked element.

To describe composition suppose we are given a list of operations $f_{\left(b_{1}, \sigma_{1}\right)}, \ldots, f_{\left(b_{k}, \sigma_{k}\right)}$ with targets $\left(y_{1}, \ldots, y_{k}\right) \in A_{t\left(b_{1}\right)} \times \ldots \times A_{t\left(b_{k}\right)}$ in $\int A$ and an operation $g_{(c, \sigma)}$ with compatible sources. Due to the equivariancy condition, we always can choose $\sigma$ in a way that these compatibility condition mean that $m_{(c . \sigma)}\left(y_{\sigma(1)}, \ldots, y_{\sigma(k)}\right)$ is the source of $g_{(c, \sigma)}$. Hence, we define the composite operation in $\int A$ as the operation $h_{(d, \pi)}$ where $d$ is an operation in $T$ obtained as a composite of $c$ and $b_{1}, \ldots, b_{k}$, the underlying morphism is the composite of two morphisms

$$
g_{(c, \sigma)} \circ m_{(c, \sigma)}\left(f_{\left(b_{1}, \sigma_{1}\right)}, \ldots, f_{\left(b_{k}, \sigma_{k}\right)}\right),
$$

and

$$
\pi=\sigma \circ\left(\sigma_{1} \times \ldots \times \sigma_{k}\right) .
$$


The unit of the monad $\int A$ sends an operation in the identity monad $(i, a)$ to the operation $i d_{\left(e_{i}, 1\right)}$ where $e_{i}=\eta(i) \in B$ and 1 is the unique function from $1 \rightarrow p^{-1}\left(e_{i}\right)$.

The polynomial monad $\int A$ comes equipped with a cartesian map of monads $\Gamma: \int A \rightarrow T$. A section of $\Gamma$ is a map of polynomial monads $T \rightarrow \int A$ such that its composite with $\Gamma$ is the identity. It is quite obvious that there is a bijection between sections and internal $T$-algebras in $A$.

We need to enhance this bijection to a functor. For this we first extend the category PMon to a 2-category PMon. Let $f, f^{\prime}: S \rightarrow T$ be two cartesian maps between polynomial monads given by the diagram

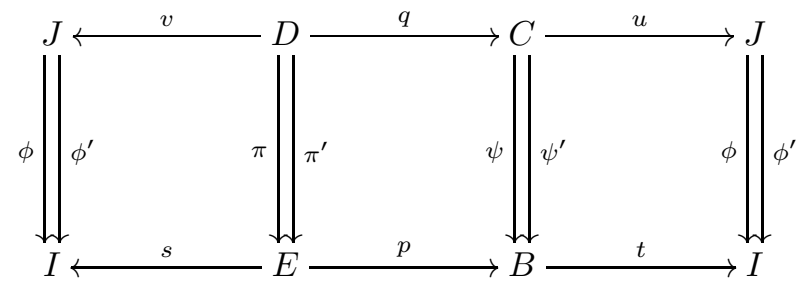

A natural transformation $\xi: f \rightarrow f^{\prime}$ consists of map $\sigma: J \rightarrow B$, such that for any $j \in J$ the set $p^{-1}(\sigma(j))$ has only one element, $t(\sigma(j))=\phi^{\prime}(j), s\left(p^{-1}(\sigma(j))\right)=\phi(j)$, and for any $c \in C$ there is an equality

$$
\mu_{T}(\sigma(u(c)) ; \psi(c))=\mu_{T}\left(\psi^{\prime}(c) ; \sigma\left(v\left(c_{1}\right)\right), \ldots, \sigma\left(v\left(c_{k}\right)\right)\right),
$$

where $\left\{c_{1}, \ldots, c_{k}\right\}=q^{-1}(c)$. If $f$ and $f^{\prime}$ are functors between small categories this definition amounts to the definition of a natural transformation between $f$ and $f^{\prime}$.

Proposition 3.3. The category of internal T-algebras in $A$ is isomorphic to the category of sections of $\Gamma$ and natural transformations between them such that their composite with $\Gamma$ is the identity natural transformation of the identity map of $T$.

Proof. By direct calculations.

Remark 3.4. If $T$ is a polynomial monad with an identity middle map (that is a small category) our polynomial Grothendick construction coincides with the classical Grothendieck construction of a functor $A: T \rightarrow$ Cat. The category of internal algebras $\operatorname{Int}_{T}(A)$ is, therefore, the lax-limit of this functor and $\int A$ is its lax-colimit.

Let PMon $/ R$ be the 2-category of polynomial monads over $R$. The objects of this 2-category are cartesian polynomial monad morphisms:

$$
g: T \rightarrow R,
$$

the morphisms are commutative triangles:

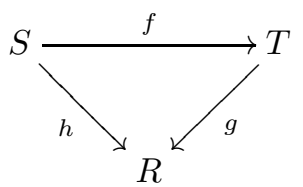


and 2-morphisms are natural transformations $f \rightarrow f^{\prime}$ such that the whiskering with $g$ is an identity transformation of $h$. With this notation, the category of sections above is the hom-category $(\mathbf{P M o n} / T)\left(T, \int A\right)$.

Now let $f: S \rightarrow T$ be a cartesian map of polynomial monads given by a commutative diagram:

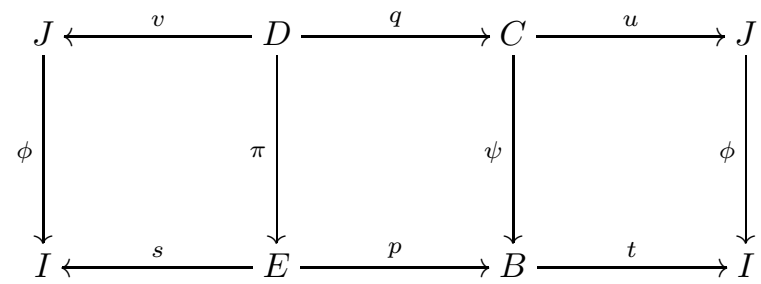

If $A$ is a categorical algebra of $T$, the algebra $f^{*}(A)$ has the following explicit description. The underlying collection of $f^{*}(A)$ is given by the collection $f^{*}(A)_{j}=$ $A_{\phi_{j}}$. The structure functor

$$
m_{(c, \sigma)}: f^{*}(A)_{v(\sigma(1))} \times \ldots \times f^{*}(A)_{v(\sigma(k))} \rightarrow f^{*}(A)_{u(c)}
$$

is given by the functor $m_{\left(\phi(c), \sigma^{\prime}\right)}$, where $\sigma^{\prime}$ is the composite

$$
\{1, \ldots, k\} \stackrel{\sigma}{\rightarrow} q^{-1}(c) \stackrel{\pi^{\prime}}{\rightarrow} \pi^{-1}(\psi(c)) .
$$

In the last display $\pi^{\prime}$ is the bijection induced by $\pi$ on fibers due to the fact that the middle square is a pullback.

Proposition 3.5. Let $A$ be a categorical algebra of a polynomial monad T. Also let $f: S \rightarrow T$ be a map of polynomial monads. Then there is a cartesian map of polynomial monads $\int f: \int f^{*}(A) \rightarrow \int A$ making the following diagram commutative

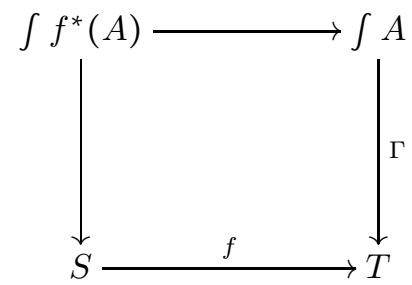

Moreover, this diagram is a pullback of polynomial monads.

Proof. The colours of $\int f^{*}(A)$ are pairs $(j, a)$ where $a \in f^{*}(A)_{j}=A_{\phi(j)}$. The operations of $\int f^{*}(A)$ are morphisms $f_{\sigma}: m_{(c, \sigma)}\left(a_{\sigma(1)}, \ldots, a_{\sigma(k)}\right) \rightarrow y$ in $f^{*}(A)_{u(c)}=$ $A_{t(\psi(c))}$, where $a_{\sigma(i)} \in f^{*}(A)_{v(\sigma(i))}=A_{\phi(v(\sigma(i)))}=A_{s(\psi(\sigma(i)))}=A_{s\left(\sigma^{\prime}(i)\right)}$. We define $\int \phi(j, a)=(\phi(j), a)$ on colours. We observe that an operation $f_{\sigma}$ as above can be interpreted as an operation $f_{\sigma^{\prime}}: m_{\psi(c), \sigma^{\prime}}\left(a_{\sigma^{\prime}(1)}, \ldots, a_{\sigma^{\prime}(k)}\right) \rightarrow y$ and, hence we define $\int \phi\left(f_{\sigma}\right)=f_{\sigma^{\prime}}$. The definition of $\int f$ on marked operations is obvious.

It is now a simple exercise to check that the square of polynomial monads is a pullback. 
We obtain the following generalisation of Proposition 3.3

Corollary 3.6. The category of internal algebras $\operatorname{Int}_{S}(A)$ of $S$ in $A$ is isomorphic to the category $(\mathbf{P M o n} / T)\left(S, \int A\right)$ of cartesian maps between polynomial monads $S \rightarrow \int A$ over $T$ and their natural transformations such that their composite with $\Gamma$ is the identity transformation of $f$.

\section{Classifiers for maps between polynomial mon- ads}

For any cartesian morphism of cartesian monads $f: S \rightarrow T$ one can associate a categorical $T$-algebra $T^{S}$ with certain universal property [3, 5, 24. Namely, this is the object representing the 2 -functor (5). This categorical $T$-algebra is called the classifier of internal $S$-algebras inside categorical $T$-algebras and is denoted $T^{S}$.

In particular, if $f=I d$ the $T$-algebra $T^{T}$ is called an absolute classifier of $T$. It was proved in 3, 5 that an absolute classifier of $T$ can be computed as a truncated simplicial $T$-algebra:

$$
\mathcal{F}_{T} 1 \underset{T \tau}{\stackrel{\mu_{1}}{\longleftarrow} \eta_{1} \Longleftarrow} \mathcal{F}_{T}(T 1) \underset{T^{2} \tau}{\stackrel{T \mu_{1}}{\longleftarrow} \mu_{T 1}} \mathcal{F}_{T}\left(T^{2} 1\right)
$$

where 1 is the terminal $I$-collection, $\tau: T(1) \rightarrow 1$ is the unique map, $\mathcal{F}_{T}$ is the free $T$-algebra functor. This simplicial object satisfies Segal's condition because $T$ is a cartesian monad and, hence, represents an internal category in the category $\operatorname{Alg}_{T}$ (Set). The last category is equivalent to $\mathrm{Alg}_{T}$ (Cat) again due to cartesianness of $T$.

It is important to understand that $T^{T}$ is a family of categories indexed by $i \in I$. It has a universal internal $T$-algebra $1 \rightarrow T^{T}$ which generates it. A component of this internal algebra $1_{i} \in\left(T^{T}\right)_{i}$ is a terminal object in this category.

Example 4.1. If $T$ is a small category, the categorical $T$-algebra $T^{T}$ is the presheaf of categories on $T$ given by comma-categories $T^{T}(i)=T / i$. The universal internal algebra is given by objects $i \stackrel{i d}{\rightarrow} i$ for each $i$.

Example 4.2. [5] For the free monoid monad Mon the absolute classifier Mon ${ }^{\text {Mon }}$ is the monoidal category of all finite ordinals $\Delta_{a l g}$. The universal internal algebra is given by the terminal ordinal [0].

Example 4.3. 5 For the free nonsymmetric operad monad NOp, the absolute classifier $N O p^{N O P}$ is the non-symmetric categorical operad of planar trees. The morphisms are generated by contractions of internal edges and introducing a single vertex on an edge. The canonical internal operad is given by the sequence of corollas.

There are analogous formulas in the non absolute case. Namely, given a cartesian map between polynomial monads $f: S \rightarrow T$ as in (6), we have the following 
commutative square of adjunctions:

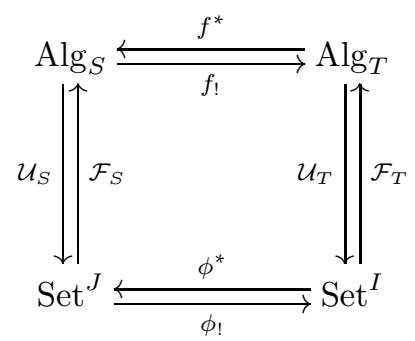

Here $\phi^{*}$ is the restriction functor $\operatorname{Set}^{I} \rightarrow \operatorname{Set}^{J}$ induced by $\phi: J \rightarrow I$ and $\phi_{!}: \operatorname{Set}^{J} \rightarrow \operatorname{Set}^{I}$ is its left adjoint given by coproducts over fibers of $\phi$.

The $T$-categorical algebra is given then by an internal categorical object similar to the absolute case:

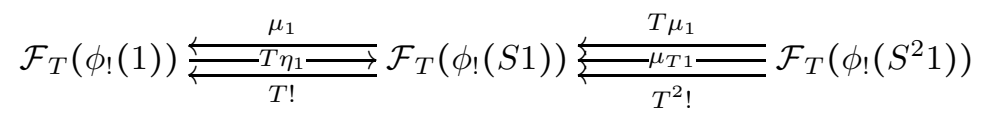

where 1 is now the terminal $J$-collection.

The classifier construction provides a 2 -functor

$$
T^{(-)}: \mathbf{P M o n} / T \rightarrow \operatorname{Alg}_{T}(\mathrm{Cat}) .
$$

Proposition 4.4. Let $T$ be a polynomial monad. The classifier 2-functor

$$
T^{(-)}: \mathbf{P M o n} / T \rightarrow A l g_{T}(\mathrm{Cat}) .
$$

is the (Cat-enriched) left adjoint to the Grothendieck construction 2-functor

$$
\int(-): \operatorname{Alg}_{T}(\text { Cat }) \rightarrow \mathbf{P M o n} / T \text {. }
$$

Proof. Let $A$ be a categorical algebra of $T$ and let $f: S \rightarrow T$ be a polynomial monad over $T$. Then

$$
\operatorname{Alg}_{T}(\mathrm{Cat})\left(T^{S}, A\right) \cong \operatorname{Int}_{S}(A) \cong \mathbf{P M o n} / T\left(S, \int A\right)
$$

by Corollary 3.6 .

Corollary 4.5. The classifier functor commutes with colimits.

In particular, given a pushout diagram of polynomial monads

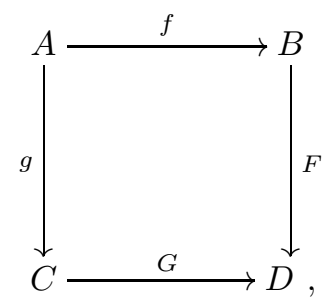


we obtain a pushout of categorical D-algebras :

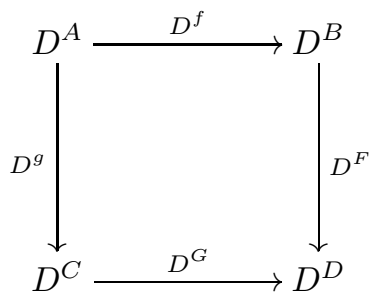

The following functorial properties of classifiers will be very useful for us:

Proposition 4.6. Let $f: S \rightarrow T$ be a map of polynomial monads. Let $f^{*}$ be the restriction functor on categorical algebras and let $f_{!}$be its left adjoint. Then

$$
f_{!}\left(S^{S}\right) \cong T^{S} .
$$

Proof. The proof is a simple exercise in universal properties of adjoints and classifiers.

This implies another functorial property of classifiers.

Proposition 4.7. Any commutative square of maps of polynomial monads

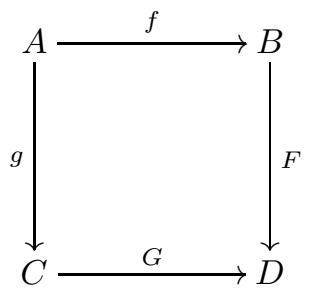

induces a map of classifiers

$$
G^{f}: C^{A} \rightarrow G^{*}\left(D^{B}\right)
$$

functorial with respect to horizontal pasting of squares.

Proof. Observe that we have a natural map of classifiers $C^{A} \rightarrow G^{*}\left(D^{A}\right)$. Indeed, by adjunction such a map corresponds to a map $G_{!}\left(C^{A}\right) \rightarrow D^{A}$. But $G_{!}\left(C^{A}\right) \cong$ $G_{!}\left(g_{!}\left(A^{A}\right)\right) \cong D^{A}$ as proved in Proposition 4.6

We can now take the composite

$$
C^{A} \rightarrow G^{*}\left(D^{A}\right) \rightarrow G^{*}\left(D^{B}\right),
$$

where the last map is induced by the upper commutative triangle of the square.

More generally given a commutative square (10) let $X$ and $Y$ be categorical algebras of $C$ and $D$ correspondingly and let $\xi: X \rightarrow G^{*}(Y)$ be its morphism. And let $x: 1 \rightarrow g^{*}(X)$ be an internal $A$-algebra in $C$ and $y: 1 \rightarrow F^{*}(Y)$ be an internal $B$-algebra in $Y$. 
Definition 4.8. A morphism of internal algebras $x \rightarrow y$ is a lax transformation $\phi: x \rightarrow f^{*}(y)$ of the internal algebras as displayed on the following square of categorical A-algebras and their morphisms:

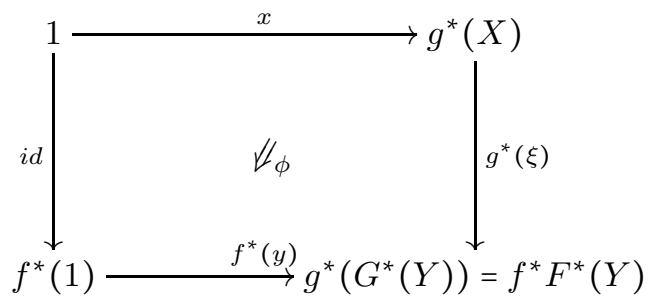

The following Proposition establishes a universal property of the morphism $G^{f}$ :

Proposition 4.9. The morphism $G^{f}$ from Proposition 4.7 induces a morphism of canonical internal algebras $a$ in $C^{A}$ and $b$ in $D^{B}$ in the sense of the Definition 4.8 such that $\phi: a \rightarrow f^{*}(b)$ is an identity and is determined by this property.

Proof. The proof is by checking universal property.

\section{$5 \quad$ Homotopy theory of algebras and classifiers}

Let $(\mathcal{E}, \otimes, e)$ be a monoidal model category and let $T$ be a polynomial monad. The category of $I$-collections $\mathcal{E}^{I}$ has a projective model structure. For a polynomial monad $T$ we can try to transfer this model structure on collections to the category of $T$-algebras along the forgetful functor $\mathcal{U}_{T}: \operatorname{Alg}_{T}(\mathcal{E}) \rightarrow \mathcal{E}^{I}$. This process requires some conditions on $\mathcal{E}$ and $T$ [5]. If we need only a semi-model model structure on $\operatorname{Alg}_{T}(\mathcal{E})$ it suffices for $\mathcal{E}$ to be cofibrantly generated 25. In many cases of interest (for simplicial sets, topological spaces or chain complexes in characteristic 0 , for example) we do have a full model structure.

Classifiers enter the scene because of the following theorem proved in [5] [Theorem 8.2].

Theorem 5.1. Let $\mathcal{E}$ be a monoidal model category with a "good" realisation functor for simplicial objects, and let $f: S \rightarrow T$ be a cartesian monad morphism between polynomial monads. Let $X$ be an $S$-algebra in $\mathcal{E}$ whose underlying $J$ collection is pointwise cofibrant. Then the I-collection underlying the left derived Quillen functor $\mathbb{L} f_{!}(X)$ can be calculated as the homotopy colimit over $T^{S}$ of the functor $\tilde{X}: T^{S} \rightarrow \mathcal{E}$ representing the $S$-algebra $X$.

The Theorem 5.1 has an important corollary which allows an interpretation of the nerve of a relative classifier of a map $f: S \rightarrow T$ as the value of the left derived functor of $f_{\text {! }}$ on the terminal $S$-algebra.

Corollary $5.2([5])$. The nerve $N\left(T^{S}\right)$ is a cofibrant simplicial T-algebra. In fact,

$$
N\left(T^{S}\right) \cong N\left(f_{!}\left(T^{T}\right)\right) \cong f_{!}\left(N\left(T^{T}\right)\right)=\mathbb{L} f_{!}(1)
$$

where 1 is the terminal simplicial $S$-algebra. 
A "good" realisation functor always exists if the category $\mathcal{E}$ is a simplicial model category. For a simplicial model category $\mathcal{M}$, we will denote by $\underline{\mathcal{M}}(X, Y)$ the simplicial hom functor between $X$ and $Y$. If $\mathcal{E}$ is a simplicial category then $\operatorname{Alg}_{T}(\mathcal{E})$ is also a simplicial category for any polynomial monad $T$. If the transferred model category structure on $\operatorname{Alg}_{T}(\mathcal{E})$ exists then it is also a simplicial model structure and an adjunction between categories of algebras generated by a map of polynomial monads is also a simplicial adjunction. In particular, all this is true for simplicial algebras of polynomial monads.

Recall also, that in a simplicial model category $\mathcal{M}$ the mapping space $\operatorname{Map}_{\mathcal{M}}(X, Y)$ can be computed as the simplicial hom $\underline{\mathcal{M}}(\operatorname{cof}(X), f i b(Y))$ where cof and $f i b$ are cofibrant and fibrant replacement respectively [15].

Theorem 5.3 (Quillen Theorem A for Polynomial monads). For any commutative diagram of maps of polynomial monads

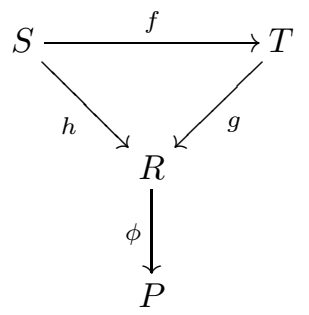

if $N\left(R^{S}\right) \rightarrow N\left(R^{T}\right)$ is a weak equivalence then $N\left(P^{S}\right) \rightarrow N\left(P^{T}\right)$ is a weak equivalence.

Proof. We have

$$
N\left(P^{T}\right) \cong(g \circ \phi) !\left(N\left(T^{T}\right)\right) \cong \phi_{!}\left(N\left(g !\left(T^{T}\right)\right) \cong \phi_{!}\left(N\left(R^{T}\right)\right) .\right.
$$

If $X$ is a fibrant simplicial $P$-algebra then the induced morphism of simplicial homs

$$
\underline{\operatorname{Alg}_{P}}\left(N\left(P^{S}\right), X\right) \leftarrow \underline{\operatorname{Alg}_{P}}\left(N\left(P^{T}\right), X\right)
$$

is isomorphic to

$$
\underline{\operatorname{Alg}_{P}}\left(\phi_{!}\left(N\left(R^{S}\right)\right), X\right) \leftarrow \underline{\operatorname{Alg}_{P}}\left(\phi_{!}\left(N\left(R^{T}\right)\right), X\right)
$$

and by adjunction to

$$
\left.\underline{\operatorname{Alg}_{R}}\left(N\left(R^{S}\right)\right), \phi^{*} X\right) \leftarrow \underline{\operatorname{Alg}_{R}}\left(N\left(R^{T}\right), \phi^{*} X\right) .
$$

Since $\phi^{*}(X)$ is fibrant and $N\left(R^{S}\right)$ and $N\left(R^{T}\right)$ are cofibrant $R$-algebra, the last map is a weak equivalence. So, $N\left(P^{S}\right) \rightarrow N\left(P^{T}\right)$ is a weak equivalence as well.

Corollary 5.4 (Classical Quillen Theorem A). If in a commutative triangle of small categories 


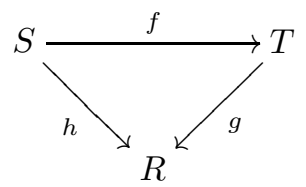

$f$ induces a weak equivalence $N(h / r) \rightarrow N(g / r)$ for any object $r \in R$ then $N(f)$ : $N(S) \rightarrow N(T)$ is a weak equivalence.

Proof. Consider this commutative triangle as commutative triangle of morphisms between polynomial monads. The maps of comma categories $h / r \rightarrow g / r$ are the components of map of classifiers $R^{S} \rightarrow R^{T}$.

Take $P=1$, the terminal category, and $\phi: R \rightarrow P$ the unique functor. Then we can apply Theorem 5.3. $N(f)$ is exactly the nerve of the map between classifiers $1^{S} \rightarrow 1^{T}$.

Corollary 5.5. Let $f: S \rightarrow T$ be a map of polynomial monads. The following statements are equivalent:

1. $N\left(T^{S}\right)$ is contractible;

2. For any commutative triangle of maps of polynomial monads

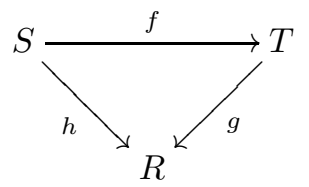

the morphism $N\left(R^{f}\right): N\left(R^{S}\right) \rightarrow N\left(R^{T}\right)$ is a weak equivalence.

Proof. (1) $\rightarrow(2)$.

The triangle above can be rewritten as a commutative diagram

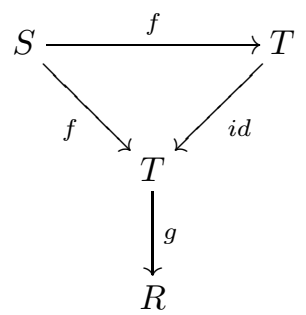

We have $N\left(T^{f}\right): N\left(T^{S}\right) \rightarrow N\left(T^{T}\right)$ is a weak equivalence since $T^{T}$ has a terminal object. By Theorem 5.3, $N\left(R^{S}\right) \rightarrow N\left(R^{T}\right)$ is a weak equivalence. $(2) \rightarrow(1)$. Take $R=T$ and $g=i d: T \rightarrow T$. Then $N\left(T^{f}\right): N\left(T^{S}\right) \rightarrow N\left(T^{T}\right)$ is a weak equivalence implies that $N\left(T^{S}\right)$ is contractible.

This result justifies the following definition. 
Definition 5.6. A cartesian map $f: S \rightarrow T$ between polynomial monads is called homotopically cofinal if $N\left(T^{S}\right)$ is contractible.

A well known classical characterisation of cofinal functors asserts that these are exactly the functors restriction along which preserves limits. We are going to provide a similar characterisation of homotopy cofinal maps between polynomial monads.

Theorem 5.7. For a commutative triangle of polynomial monads (12) the following statements are equivalent:

1. The map $N\left(R^{f}\right): N\left(R^{S}\right) \rightarrow N\left(R^{T}\right)$ is a weak equivalence.

2. For any simplicial $R$-algebra $X$ the morphism $f$ induces a weak equivalence of homotopy mapping spaces

$$
\operatorname{Map}_{\operatorname{Alg}_{S}}\left(1, h^{*} X\right) \rightarrow \operatorname{Map}_{\operatorname{Alg}_{T}}\left(1, g^{*} X\right) .
$$

Here 1 means the terminal simplicial algebra.

Proof. From the beginning we can assume that $X$ is a fibrant $R$-algebra. We can compute the mapping space $\operatorname{Map}_{\operatorname{Alg}_{S}}\left(1, h^{*} X\right)$ as the simplicial hom $\operatorname{Alg}_{S}\left(\operatorname{cof}(1), h^{*} X\right)$ where $\operatorname{cof}(1)$ is a cofibrant replacement for the terminal algebra 1. By adjunction this space is isomorphic to $\underline{A l g_{R}}\left(\mathbb{L} h_{!}(1), X\right)$. By Corollary $5.2 \mathbb{L} h_{!}(1) \cong N\left(R^{S}\right)$. Then

Similarly

$$
\underline{\operatorname{Alg}_{R}}\left(\mathbb{L} h_{!}(1), X\right) \sim \underline{\operatorname{Alg}_{R}}\left(N\left(R^{S}\right), X\right)
$$

$$
\underline{\operatorname{Alg}_{R}}\left(g_{!}(\operatorname{cof}(1)), X\right) \sim \underline{\operatorname{Alg}_{R}}\left(N\left(R^{T}\right), X\right) .
$$

Hence, $N\left(R^{f}\right)$ induces a weak equivalence between these simplicial sets.

It is obvious that we can reverse these calculations and so prove that $N\left(R^{f}\right)$ is a weak equivalence provided it induces a weak equivalence between mapping spaces for all $R$-algebra $X$.

Corollary 5.8. A cartesian map between polynomial monads $f: S \rightarrow T$ is homotopically cofinal if and only if for any simplicial $T$-algebra $X$ it induces a weak equivalence between mapping spaces:

$$
M a p_{\operatorname{Alg}_{S}}\left(1, f^{\star} X\right) \rightarrow M a p_{\operatorname{Alg}_{T}}(1, X) .
$$

Recall that homotopy left cofinal functor is defined in [15][Definition 19.6.1] as a functor between small categories $f: S \rightarrow T$ such that $N(f / i)$ is contractible for all objects $i \in I$. It coincides with our notion of homotopically cofinal map between polynomial monads when the latter is specialised to small categories.

Corollary 5.9 (Theorem 19.6.13(b) [15]). A functor $f: S \rightarrow T$ between small categories is homotopically left cofinal if and only if for any functor $X: T \rightarrow$ SSet it induces a weak equivalence

$$
\operatorname{holim}_{S} f^{*} X \rightarrow \operatorname{holim}_{T} X .
$$


Proof. The homotopy limit of a functor $X$ can be computed as a mapping space from terminal functor to $X$.

Remark 5.10. This theorem (and its dual) is proved in [15] in a slightly more general setting. We are not going to pursue this generality in this paper but it is not hard to get Hirschhorn's Theorem from the corollary above and the model theoretic argument from [5].

Remark 5.11. If $\mathcal{W}$ is a fundamental localizer of Grothendieck 8 then all the results of this section can be localized with respect to $\mathcal{W}$. In particular, if we take $\mathcal{W}=\mathcal{W}_{0}$ (so the weak equivalences between small categories become functors which induce isomorphisms on $\pi_{0}$ ) the notion of $\mathcal{W}_{0}$-homotopically cofinal functor coincides with the classical categorical notion of cofinal functor.

Remark 5.12. In [1] the first author used the fact that the map of polynomial monads $O p_{n} \rightarrow S O p$ is a $\mathcal{W}_{n-2}$-homotopically cofinal functor to prove the BaezDolan stabilization hypothesis for Rezk's weak $n$-categories. Here, $O p_{n}$ is the polynomial monads for $n$-operads, $S O p$ is the polynomial monad for symmetric operads and $\mathcal{W}_{n-2}$ is the fundamental localizer for $(n-2)$-truncated homotopy type.

\section{Twisted Boadrman-Vogt tensor product and Thomason's theorem}

Since polynomial monads form a 2-category we can ask about lax-colimits of a diagram in PMon. Such a lax-colimit can be given explicitly as another version of Grothendieck construction.

Let $A$ be a small category and $F: A \rightarrow$ PMon be a strict 2-functor. We then define a new polynomial monad $\oint F$ as follows. The set of its colours is the set of pairs $(a, i)$ where $a \in A$ and $i \in F(a)_{0}$ is a colour of the polynomial monad $F(a)$. An operation consists of the following data:

1. An operation $\beta \in F(b)$

2. A family of morphisms $\alpha_{d}: a_{d} \rightarrow b$ in $A$, where $d$ runs over the set $p_{b}^{-1}(\beta)$ and where $p_{b}$ is the middle map of the polynomial monad $F(b)$;

3. A family of colours of $j_{d} \in F\left(a_{d}\right), d \in p^{-1}(\beta)$ such that $F \alpha_{d}\left(j_{d}\right)=s_{b}(d), d \in$ $p_{b}^{-1}(\beta)$, where $s_{b}$ is the source map of the monad $F(b)$.

A marked operation is the operation $\beta$ with a marked element $d \in p^{-1}(\beta)$ and the middle map forgets the marking. The source map assigns to a marked operation the pair $\left(a_{d}, j_{d}\right)$, where $d$ is the marked element. The target of an operation $\beta$ as above is the pair $(b, t(\beta))$. The unit and composition operation of the polynomial monad $\oint F$ are obvious now. In order to distinguish this construction from Grothendieck construction of a categorical algrebra we will call the polynomial monad $\oint F$ twisted Boardman-Vogt tensor product.

A relation of twisted Boardman-Vogt product with the classical Grothendieck construction is given by the following: 
Proposition 6.1. Let $\mathcal{E}$ be a symmetric monoidal category. The category of algebras of $\oint F$ is isomorphic to the category of sections of the Grothendieck construction of the functor

$$
A l g_{F}(\mathcal{E}): A^{o p} \rightarrow C A T
$$

which associates to an $a \in A$ the category $\operatorname{Alg}_{F(a)}(\mathcal{E})$ and to a morphism $f: a \rightarrow b$ the functor $F(f)^{*}: \operatorname{Alg}_{F(b)}(\mathcal{E}) \rightarrow \operatorname{Alg}_{F(a)}(\mathcal{E})$.

That is an algebra $X$ of $\oint F$ consists of a family of $F(a)$-algebras $X(a), a \in A$ together with family of $F(a)$-morphisms $\phi(a): X(a) \rightarrow F(f)^{*} X(b)$ for each $f$ : $a \rightarrow b$ which satisfies obvious functoriality conditions.

Proof. By direct inspection.

The following corollary justifies our terminology.

Corollary 6.2. Let $D$ be a polynomial monad and let $F: A \rightarrow$ PMon be the constant functor $F(a)=D$ then

$$
\oint F \cong D \otimes_{B V} A
$$

where $\otimes_{B V}$ is the Boardman-Vogt tensor product of symmetric operads.

Proof. Indeed, algebras of $\oint F$ in this case are just presheaves of algebras of $D$, which is a defining property of the Boardman-Vogt tensor product.

Remark 6.3. The Boardman-Vogt product was defined for symmetric operads not for polynomial monads. It can not be restricted under equivalence between $\Sigma$ free symmetric operads and polynomial monads in general because the result of this product may not be $\Sigma$-free. Famous example is, of course, the isomorphism $A s s \otimes_{B V} A s s \cong C o m$, which is an incarnation of the Eckmann-Hilton argument.

But, it is not hard to check that the $B V$-product of a $\Sigma$-free operad and a category (symmetric operad with unary operations only) is again $\Sigma$-free, so our sentence makes sense.

Corollary 6.4. Let $X$ be a categorical algebra of $\oint F$. Then an internal $\oint F$ algebra $x$ in $X$ consists of a family of internal $F(a)$-algebras $x(a)$ in $X(a)$ together with a family of morphisms of internal algebras (in the sense of Definition 4.8) $\phi(a): x(a) \rightarrow f^{*}(x(b))$, which satisfies the usual lax-compatibility conditions.

It is obvious that in the case of presheaves in Cat twisted $B V$-product and Grothendieck constructions are equivalent. Indeed, given $F: A \rightarrow$ Cat one can form $\oint F$. But we also can consider $F$ as a categorical $A$-algebra. Then $\int F$ is isomorphic to $\oint F$. But in general, there can not be even a cartesian polynomial monad map $\oint F \rightarrow A$ because if such a map exists the pullback of the middle maps would force $\oint F$ to be a category. Instead of this we have an obvious functoriality of the twisted $B V$-product in the sense that every natural transformation $\psi: F \rightarrow G$ of presheaves of polynomial monads over $A$ induces a map of polynomial monads

$$
\oint \psi: \oint F \rightarrow \oint G
$$


If $F$ is a presheaf of small categories and 1 is the constant terminal presheaf of small categories then the unique morphism $e: F \rightarrow 1$ induces $\oint e: \oint F \cong \int F \rightarrow \oint 1=A$, which is exactly the projection from classical Grothendieck construction.

Given $\psi: F \rightarrow G$ we can now construct the classifier $(\oint G)^{\phi F}$.

Proposition 6.5. The classifier $(\oint G)^{\oint F}$ is the categorical $\oint G$-algebra freely generated by the internal $F(a)$ algebras $x(a) \in G(a)^{F(a)}, a \in A$ together with morphisms $\phi(a): x(a) \rightarrow f^{*}(x(b))$ for each $f: a \rightarrow b$ in $A$ which satisfy obvious lax-functorial property.

Proof. This follows from general description of universal properties of the classifiers and Lemma 6.4.

Given $\psi: F \rightarrow G$ we also can construct yet another canonical categorical $\oint G$ algebra as follows. For each $a \in A$ we take the classifier $G(a)^{F(a)}$ of $\psi(a): F(a) \rightarrow$ $G(a)$. By Proposition 4.7 these objects form an algebra in Cat of $\oint$ G. By slightly abusing notations we will call it $G^{F}$ and call it local classifier algebra of $\psi$.

Theorem 6.6. There is a canonical morphism

$$
\Psi:(\oint G)^{\oint F} \rightarrow G^{F}
$$

of categorical $\oint G$-algebras which is a weak equivalence of simplicial $\oint G$-algebras after application of nerve functor.

Proof. We can get the map $\Psi$ from universal property of classifiers as follows. It will be enough to observe that $G^{F}$ contains a canonical internal $\oint F$-algebra. But this follows from Proposition 4.9 and Corollary 6.4 where all morphisms $\phi(a)$ are identities.

Also observe that according to Proposition 6.5 each $G(a)$-algebra $(\oint G)^{\oint F}(a)$ contains an internal $F(a)$-algebra which immediately provides us with a $G(a)$ algebra morphism $p(a): G^{F}(a) \rightarrow(\oint G)^{\oint F}(a)$. By universal property the functor $p(a)$ is a section of $\Psi(a)$ but not necessary a $(\oint G)^{\oint F}$-algebras morphism. But we claim $p(a)$ is the left adjoint to $\Psi(a)$. Indeed the counit $p(a)\left(\psi(a)\left(f^{*}(b)\right) \rightarrow\right.$ $f^{*}(x(b))$ on the generating internal $F(b)$-algebra $x(b)$ in in $G(b)$ is given by canonical morphism $\phi(a): x(a) \rightarrow f^{*}(x(b))$ since $p(a)\left(\psi(a)\left(f^{*}(b)\right)=x(a)\right.$ for any $f: a \rightarrow b$ (since the morphism $\Psi$ maps each $\phi(a)$ to the identity).

Remark 6.7. This theorem allows to see more relations between twisted $B V$ product and Grothendieck construction. There is a canonical factorization of the map of polynomial monads $\oint \psi: \oint F \rightarrow \oint G$ :

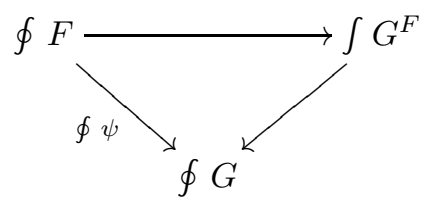


This is just the mate under the adjuction between Grothendieck construction and classifier functor of the canonical map $\Psi$ from the classifier $(\oint G)^{\phi F}$ to the local classifier algebra $G^{F}$.

In the case of a map of presheaves in Cat the canonical morphism $\Psi$ can be also understood as the counit of the adjunction between Grothendieck construction and classifier functor.

Corollary 6.8. The simplicial $\oint G$-algebra $N\left((\oint G)^{\oint F}\right)$ is a cofibrant replacement of the simplicial $\oint$ G-algebra $N\left(G^{F}\right)$.

Now let $\psi: F \rightarrow G$ be a morphism of presheaves of polynomial monads over a polynomial monad $D$. By universal property of lax-colimit we then have a commutative triangle of maps of polynomial monads:

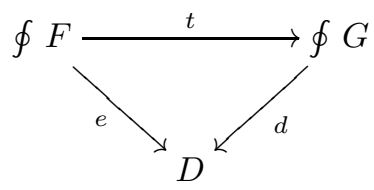

where $t=\oint \psi$. The map $d$ induces a left Quillen functor $d_{!}: A l g_{\oint}(S S e t) \rightarrow$ $\operatorname{Alg}_{D}($ SSet $)$.

Proposition 6.9. There exists an isomorphism in the homotopy category of simplicial D-algebras:

$$
\mathbb{L} d_{!}\left(N\left(G^{F}\right)\right) \cong N\left(D^{\oint F}\right) .
$$

Proof. Compute

$$
e_{!}\left(N\left((\oint F)^{\oint F}\right)=N\left(e_{!}\left((\oint F)^{\oint F}\right)=N\left(D^{\oint F}\right) .\right.\right.
$$

On the other hand this object is equal to

$$
d_{!} t_{!}\left(N\left((\oint F)^{\oint F}\right)=d_{!}\left(N t_{!}\left((\oint F)^{\oint F}\right)\right)=d_{!}\left(N\left((\oint G)^{\oint F}\right) .\right.\right.
$$

Since $N\left((\oint G)^{\oint F}\right.$ is a cofibrant replacement for the nerve of $G^{F}$ we have that the last object is isomorphic in $H o\left(\operatorname{Alg}_{D}(S S e t)\right.$ to the result of application of the derived functor of $d_{!}$to $N\left(G^{F}\right)$.

This Proposition allows us to prove the following generalisation of a classical Thomason's theorem about homotopy colimits of small categories [21].

Theorem 6.10. Let $F$ be a presheaf of polynomial monads over a polynomial monad D. Then $N\left(D^{\phi F}\right)$ is weakly equivalent to hocolim ${ }_{A} N\left(D^{F(a)}\right)$.

Proof. We take $G$ to be the constant functor $G(a)=D$. Then we have a natural transformation of presheaves $\psi: F \rightarrow G$ whose components are derived from the components of cocone of $F$ over $D$. The left adjoint $d_{!}$is then the colimit over $A$ in the category of $D$-algebras. 
Corollary 6.11 (Thomason). For a presheaf $F$ of small categories over $A$ the nerve of its Grothendieck construction is homotopy equivalent to the homotopy colimit of the presheaf of nerves.

Proof. It is sufficient to take $D=1$. The classifier $1^{\phi F}$ is just the Grothendieck construction $\int F$ and the local classifier algebra $1^{F}$ is just $F$ itself.

Theorem 6.12. Let $F$ be a presheaf of polynomial monads over a polynomial monad D. The following conditions are equivalent

1. $\oint F \rightarrow D$ is a homotopically cofinal functor;

2. $N\left(D^{\oint F}\right)$ is contractible;

3. For any map of polynomial monads $D \rightarrow R$ the natural map

$$
\operatorname{hocolim}_{A} N\left(R^{F(a)}\right) \rightarrow N\left(R^{D}\right)
$$

is a weak equivalence.

Proof. The equivalence of (1) and (2) is the definition of homotopically cofinal functor. Now (2) is equivalent to (3) by Theorem 6.10 and Corollary 5.5.

\section{Homotopy pushouts of classifiers}

For our application purpose we will be mostly interested in homotopy pushouts of classifiers. In other words we are going to consider presheaves of polynomial monads over the category $\Lambda$ with three objects and two nontrivial arrows:

$$
2 \longleftarrow 0 \longrightarrow 1
$$

Main question for us is then given a commutative square of polynomial monads (10) over a polynomial monad $R$ when the square

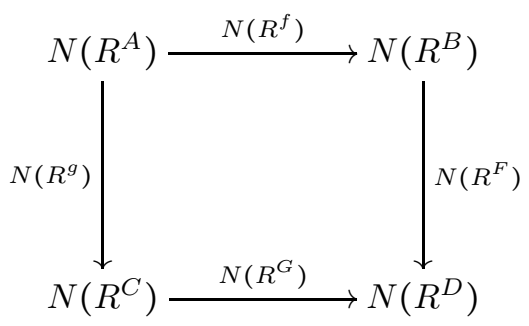

is a homotopy pushout square in the category of $R$-algebras?

Example 7.1. In the square above let $F: \Lambda \rightarrow \mathbf{P M o n}$ is such that $F(0)=A, F(1)=$ $B, F(2)=C$ are monoids and $D$ be the pushout of this diagram. Let $R=1$. Then the map hocolim ${ }_{\Lambda} N\left(R^{F(a)}\right) \rightarrow N\left(R^{D}\right)$ is the map from homotopy pushout of classifying spaces of this diagram of monoids to the classifying space of the 
pushout. This situation was considered by Fiedorowicz in [12, Theorem 4.1]1. It was proved that a sufficient condition for this map to be a weak equivalence is that $\mathbb{Z}[B]$ and $\mathbb{Z}[C]$ are flat $\mathbb{Z}[A]$-modules, where $\mathbb{Z}[M]$ is the monoid ring of a monoid $M$.

An example away from categories is one of the main topics in [5]:

Example 7.2. Let $T$ be a polynomial monad with the set of colors $C$ and let $F(1)=T$ and $F(0)=F(2)=I$, where $I$ is the identity polynomial monad with the same set of colours $C$. The colimit of such diagram is $T=D$ again. The monad $\oint F$ in this case is the monad $T^{f, g}$ from [5]. Let $R=T$ then the colimit over the classifier $T^{T^{f, g}}$ 'computes' the pushouts of algebras of $T$ along a free map of $T$-algebras. The homotopy type of $N\left(T^{T^{f, g}}\right)$ can be very nontrival.

Definition 7.3. We will call a commutative square of polynomial monads (10) homotopically cofinal if the equivalent conditions of Theorem 6.12 are satisfied.

Thus for a homotopically cofinal square any square like (13) is a homotopy pushout of $R$-algebras.

Proposition 7.4. 1. For any polynomial monad $D$ the constant square

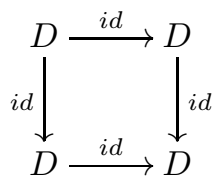

is homotopically cofinal.

2. If in a commutative diagram of polynomial monads

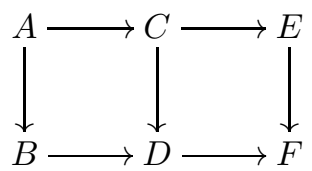

the left square is homotopically cofinal, then the outer square is homotopically cofinal if and only if the right square is homotopically cofinal.

3. In a commutative cube of polynomial monads

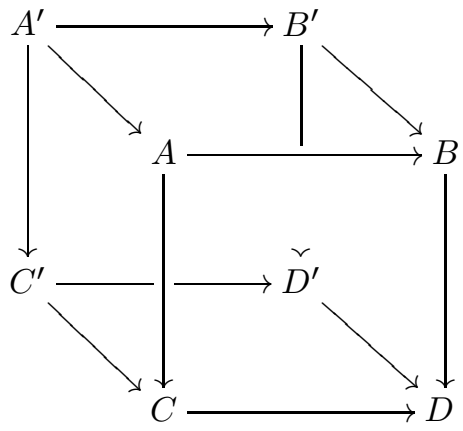

${ }^{1}$ First author is grateful to Andrey Lazarev for pointing out to this Fiedorowicz's theorem. 
let the back square be homotopically cofinal and let $A^{\prime} \rightarrow A, B^{\prime} \rightarrow B$ and $C^{\prime} \rightarrow$ $C$ homotopically cofinal morphisms. Then the front square is a homotopically cofinal square if and only if $D^{\prime} \rightarrow D$ is homotopy cofinal.

Proof. For the first statement we observe similarly that for any $D \rightarrow R$ we get a constant square of nerves of classifiers which is a homotopy pushout in the category of simplicial $R$-algebras.

To prove the second statement it is enough to consider an arbitrary map of polynomial monads $F \rightarrow R$. Then we obtain a commutative diagram of classifiers over $R$. From standard properties of homotopy pushouts we get that after application of nerve the outer square is a homotopy pushout of simplicial $R$-algebras if and only if the right square is a homotopy pushout. So Theorem 6.12 implies the result.

Finally, for the third statement let $F: \Lambda \rightarrow$ PMon be the functor which constitutes the left corner of the front square. Let $F^{\prime}: \Lambda \rightarrow$ PMon be the corresponding functor for the back square. Then we have a commutative square of polynomial monads maps:

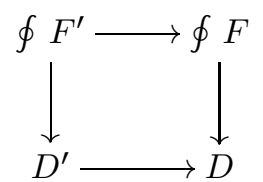

The nerve of the classifier of the map $\oint F^{\prime} \rightarrow \oint F$ is the cofibrant replacement of the nerve of the local classifier $F^{F^{\prime}}$ which is contractible because $F(i) \rightarrow F^{\prime}(i)$ is homotopically cofinal for each $i=0,1,2$. So, by Corollary 5.5 the map

$$
N\left(D^{\oint F^{\prime}}\right) \rightarrow N\left(D^{\oint F}\right)
$$

is a weak equivalence. On the other hand, since the back square is homotopically cofinal $\oint F^{\prime} \rightarrow D^{\prime}$ is a homotopically cofinal map by definition. Therefore, the map

$$
N\left(D^{\oint F^{\prime}}\right) \rightarrow N\left(D^{D^{\prime}}\right)
$$

is also a weak equivalence. The composite of two homotopically cofinal maps is homotopically cofinal map again and so if one of the two maps $D^{\prime} \rightarrow D$ or $\oint F \rightarrow D$ is homotopically cofinal, $\oint F^{\prime} \rightarrow D$ is homotopically cofinal and the nerve of its classifier is contractible which implies that the other map must also be homotopically cofinal.

Remark 7.5. It is instructive to give a description of the classifier $D^{\oint F}$, for $F$ : $\Lambda \rightarrow$ Poly as in the square (10). According to general theory of classifiers from [5, Section 6.3] an object of this categorical $D$-algebra are given by specifying of

1. an operation $\beta$ of the polynomial monad $D$;

2. a labelling of the sources of $\beta$ by numbers $0,1,2$. 
Morphisms are generated by morphisms in $D^{A}$, when we multiply operations in $D$ with 0-labelled sources and, similarly, by morphisms in $D^{B}$ and $D^{C}$ when we multiply operations with 1 and 2 labelled sources respectively. We also have two other type of generators: one can replace a source labelled by 0 by a source labelled by 1 or 2 . There are relations on this type of morphisms which comes from the requirement that $f$ and $g$ in (10) are polynomial monad maps.

\section{Mapping spaces between pointed algebras of polynomial monads}

Let $T$ be a polynomial monad and let $T_{*}$ be the monad whose category of algebras is the category of pointed $T$-algebras; that is, the comma-category $1 / \mathrm{Alg}_{T}$. There is a map of monads

$$
u: T \rightarrow T_{*}
$$

such that the restriction functor $u^{*}: \operatorname{Alg}_{T_{*}} \rightarrow \operatorname{Alg}_{T}$ 'forgets the point'. Analogously, let $T_{* *}$ be the category of double pointed algebras; that is, the category $1 \amalg 1 / \mathrm{Alg}_{T}$. We have a pushout of monads:

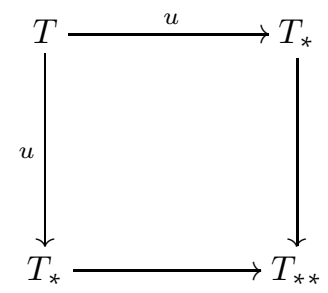

We can consider this pushout as a pushout of monads over $T_{\star}$ because the identity $i d: T_{*} \rightarrow T_{*}$ induces a map of monads

$$
U: T_{* *} \rightarrow T_{*} .
$$

such that the restriction functor $\mathrm{Alg}_{T_{\star}} \rightarrow \mathrm{Alg}_{T_{* *}}$ 'doubles the point'.

Theorem 8.1 (Formal delooping Theorem). Assume $T_{*}$ is a polynomial monad and the square (14) is homotopically cofinal. Then, for any pointed simplicial $T$-algebra $X$, there is a weak equivalence of simplicial sets:

$$
\Omega M a \operatorname{Alg}_{T}\left(1, u^{*} X\right) \sim \operatorname{Map}_{\operatorname{Alg}_{T * *}}\left(1, U^{*} X\right)
$$

where $\Omega \operatorname{Map}_{\mathrm{Alg}_{T}}\left(1, u^{*} X\right)$ is the loop space with the base point given by the point $1 \rightarrow X$ in the $T$-algebra $X$.

Proof. By assumption the square (14) satisfies the conditions of Theorem 6.12, Therefore we have a homotopy pushout of nerves of classifiers over $T_{\star}$. For a 
fibrant replacement $f i b(X)$ of a $T_{*}$-algebra $X$, we then have a homotopy pullback

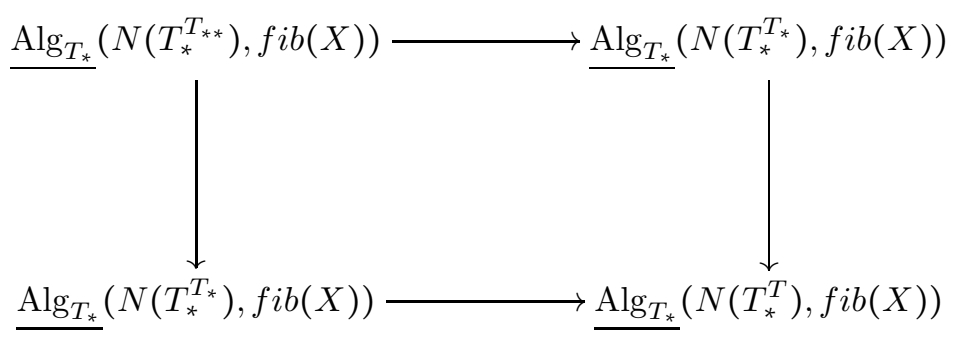

By adjunctions

$$
\begin{gathered}
\operatorname{Alg}_{T_{*}}\left(N\left(T_{*}^{T_{* *}}\right), f i b(X)\right) \sim \operatorname{Map}_{\mathrm{Alg}_{T_{* *}}}\left(1, U^{*} X\right), \\
\underline{\operatorname{Alg}_{T_{*}}}\left(N\left(T_{*}^{T}\right), f i b(X)\right) \sim \operatorname{Map}_{\operatorname{Alg}_{T}}\left(1, u^{*} X\right)
\end{gathered}
$$

and

$$
\underline{\operatorname{Alg}_{T_{*}}}\left(N\left(T_{*}^{T_{*}}\right), f i b(X)\right) \sim \operatorname{Map}_{\mathrm{Alg}_{T_{*}}}(1, X) .
$$

But, in the category of pointed $T$-algebras, the terminal algebra is also the initial object so the space $\operatorname{Map}_{\mathrm{Alg}_{T_{*}}}(1, X)$ is contractible. This completes the proof.

Remark 8.2. The condition that the monad of pointed algebras is polynomial is not trivial. This is true for any tame monad in the sense of Batanin and Berger [5. Yet, for example, it does not hold for the monad for symmetric operads.

Let $T$ be a polynomial monad with set of colours $I$ and let $i \in I$. Let $I d$ be the identity polynomial monad on Set, and let $I d_{I}$ be the identity polynomial monad on $\operatorname{Set}^{I}$. There is a cartesian map of polynomial monads

$$
i: I d \rightarrow I d_{I}
$$

which sends the unique colour to the element $i$ and the unique operation to the identity on $i$. (Both monads are small categories, the map $i$ corresponds to the functor from the terminal category which picks up the object $i$.)

We also have a one coloured polynomial monad $I d_{+}$which 'adds a point' to each set $X$. Explicitly, $I d_{+}$is given by the following polynomial

$$
1 \longleftrightarrow \stackrel{s}{\longrightarrow} 2 \stackrel{t}{\longrightarrow} 1
$$

where 2 is the set with two elements $\{0,1\}$ and $p$ sends 1 to 0 . The algebras of $I d_{+}$ are pointed sets. Let now $T_{+}$be the pushout of polynomial monads

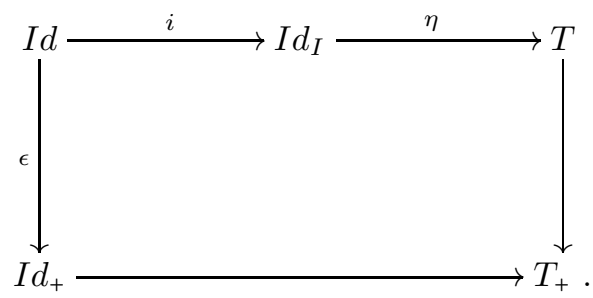


The algebras of $T_{+}$are, therefore, the algebras of $T$ equipped with a marked point in degree $i \in I$. We called them $i$-pointed $T$-algebras.

Now let

$$
f: T \rightarrow S
$$

be a map of polynomial monads. Suppose that the composite $i \circ \eta \circ f: I d \rightarrow S$ can be factorised through the unit of $I d_{+}$. We then have a map of polynomial monads

$$
F: T_{+} \rightarrow S
$$

This factorisation condition says that the $\phi(i)$-th component $X_{\phi(i)}$ of any $S$ algebra $X$ has a 'marked' point $1 \rightarrow X_{i}$ and the restriction functor $f^{*}$ preserves this canonical point.

Theorem 8.3 (Formal Fibration sequence Theorem). If the square (15) is homotopically cofinal then for any simplicial $S$-algebra $X$, there is a fibration sequence

$$
\operatorname{Map}_{\operatorname{Alg}_{T_{+}}}\left(1, F^{*} X\right) \rightarrow \operatorname{Map}_{\operatorname{Alg}_{T}}\left(1, f^{*} X\right) \rightarrow f i b\left(X_{i}\right),
$$

where $f i b\left(X_{i}\right)$ is a fibrant replacement for the simplicial set $X_{i}$.

Proof. Let $f i b(X)$ be the fibrant replacement of the $S$-algebra $X$. Observe then, that the $i$-th component $f i b(X)_{i}$ is also a fibrant replacement $f i b\left(X_{i}\right)$ for the simplicial set $X_{i}$. We have the following homotopy pushout of nerves of classifiers

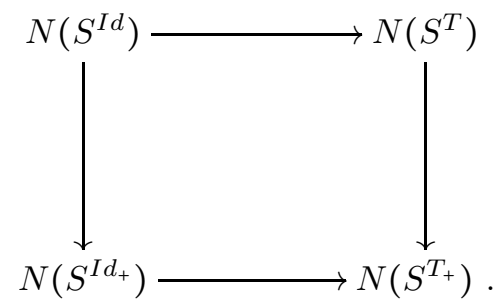

We then have a homotopy pullback of simplicial sets

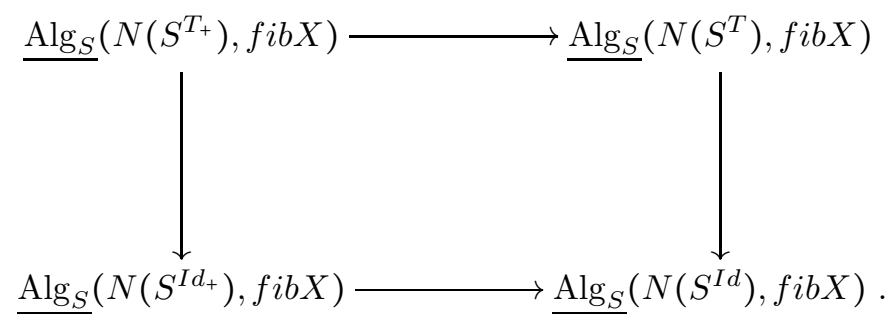

By adjunction we have a simplicial set isomorphism

$$
\underline{\operatorname{Alg}_{S}}\left(N\left(S^{I d}\right), f i b X\right) \cong \underline{\operatorname{Alg}_{I d}}\left(N\left(I d^{I d}\right), i^{*} \eta^{*} f^{*}(f i b X)\right) .
$$


The space $i^{*} \eta^{*} f^{*}(f i b X)$ is just the simplicial set $f i b\left(X_{i}\right)$ and we have

$$
\underline{\operatorname{Alg}_{I d}}\left(N\left(I d^{I d}\right), i^{*} \eta^{*} f^{*}(f i b X)\right) \cong \operatorname{Map}_{\operatorname{Alg}_{I d}}\left(1, f i b\left(X_{i}\right)\right) \sim f i b\left(X_{i}\right) .
$$

Analogously:

$$
\underline{\operatorname{Alg}_{S}}\left(N\left(S^{I d_{+}}\right), f i b X\right) \cong \underline{\operatorname{Alg}_{I d_{+}}}\left(N\left(I d_{+}^{I d_{+}}\right), \alpha^{*}(f i b X)\right)
$$

where $\alpha: I d_{+} \rightarrow S$. It can be seen by a direct calculation or by the universal property that the classifier $I d_{+}^{I d_{+}}$is just the pointed category with two objects 0 (a point) and 1 , and one nontrivial arrow $0 \rightarrow 1$. The nerve of this category is a pointed simplicial interval. Hence, $\operatorname{Alg}_{I d_{+}}\left(N\left(I d_{+}^{I d_{+}}\right), \alpha^{*}(f i b X)\right)$ is the path space over $f i b\left(X_{i}\right)$.

These calculations show that the bottom arrow in the homotopy pullback (16) is the classical path fibration over $f i b\left(X_{i}\right)$. The rest of the proof follows from the usual adjunction argument.

Remark 8.4. A special case of this Theorem is when $F$ is an identity map. Then, for any $i$-pointed $T$-algebra, we have a fibration sequence

$$
M a \operatorname{Malg}_{T_{+}}(1, X) \rightarrow M a p_{\operatorname{Alg}_{T}}(1, X) \rightarrow f i b\left(X_{i}\right),
$$

where we skip notation for the forgetful functor from $i$-pointed $T$-algebras to $T$ algebras. This is a conceptual explanation of Theorem 8.3

\section{Part II}

\section{Applications}

\section{Multiplicative operads, bimodules and weak bi- modules}

The non-symmetric operad which is equal to the unit $e$ in each degree is called associativity operad and is denoted by Ass. If $\mathcal{E}$ is a cartesian category then $A s s=1$ is the terminal object in the category of non-symmetric operads NOp.

Remark 9.1. In the literature, Ass is often used to denote the symmetrised version of our associative operad.

Definition 9.2. A multiplicative non-symmetric operad is a non-symmetric operad $\mathcal{O}$ together with an operadic morphism

$$
A s s \rightarrow \mathcal{O}
$$

The category of multiplicative non-symmetric operads $\mathrm{NOp}_{*}$ is the category Ass/NOp. We have a forgetful functor

$$
u^{*}: \mathrm{NOp}_{*} \rightarrow \mathrm{NOp}
$$


Definition 9.3. Let $\mathcal{A}$ and $\mathcal{C}$ be two non-symmetric operads. An $\mathcal{A}-\mathcal{C}$-bimodule in a symmetric monoidal category $\mathcal{E}$ is given by

- an object $\mathcal{B}_{n}$ for all $n \geq 0$

- morphisms

$$
\mathcal{A}_{k} \otimes \mathcal{B}_{n_{1}} \otimes \ldots \otimes \mathcal{B}_{n_{k}} \rightarrow \mathcal{B}_{n_{1}+\ldots+n_{k}}
$$

called left actions

- morphisms

$$
\mathcal{B}_{k} \otimes \mathcal{C}_{n_{1}} \otimes \ldots \otimes \mathcal{C}_{n_{k}} \rightarrow \mathcal{B}_{n_{1}+\ldots+n_{k}}
$$

called right actions

satisfying the obvious analogue of the axioms for non-symmetric operad and a compatibility condition between left and right actions.

Remark 9.4. Notice that in the definition of left action one can take $k=0$ and so an empty product of $\mathcal{B}_{\mathrm{s}}$; that is, the tensor unit $e \in \mathcal{E}$. On the right hand side we will have then an empty sum of natural numbers that is 0 . So we have a map $\mathcal{A}_{0} \rightarrow \mathcal{B}_{0}$ as one of the structure operations for the left $\mathcal{A}$-module.

Remark 9.5. It is not hard to see that the left Ass-module on a family $\mathcal{B}_{n} \in \mathcal{E}$ is given by an associative pairing:

$$
\mathcal{B}_{p} \times \mathcal{B}_{q} \rightarrow \mathcal{B}_{p+q}
$$

which is unital with respect to the unit $A s s_{0} \rightarrow \mathcal{B}_{0}$.

The right $A s s$-module structure is the same as a structure of a functor $\mathcal{B}_{n}=$ $\mathcal{B}([n])$ on the subcategory $\Delta_{\text {surj }} \subset \Delta$ of order-preserving surjections. For the $A s s$-Ass-bimodule these structures are required to be compatible in the obvious sense.

Recall that non-symmetric operads can be defined in terms of $\circ_{i}$-operations [18, Definition 11]. The $\circ_{i}$-operations are obtained as the composites :

$\mathcal{O}_{k} \otimes \mathcal{O}_{n}=\mathcal{O}_{k} \otimes e \otimes \ldots \otimes \mathcal{O}_{n} \otimes \ldots \otimes e \stackrel{1 \otimes \epsilon \otimes \ldots \otimes 1 \otimes \ldots \otimes \epsilon}{\longrightarrow} \mathcal{O}_{k} \otimes \mathcal{O}_{1} \otimes \ldots \otimes \mathcal{O}_{n} \otimes \ldots \otimes \mathcal{O}_{1} \stackrel{m}{\longrightarrow} \mathcal{O}_{k+n-1}$

Definition 9.6. Let $\mathcal{A}$ and $\mathcal{C}$ be two non-symmetric operads. $A$ weak $\mathcal{A}-\mathcal{C}$ bimodule $\mathcal{W}$ in a symmetric monoidal category $\mathcal{E}$ is given by

- an object $\mathcal{W}_{n}$ in $\mathcal{E}$ for all $n \geq 0$

- for $i=1, \ldots, k$, morphisms

$$
\circ_{i}: \mathcal{A}_{k} \otimes \mathcal{W}_{n} \rightarrow \mathcal{W}_{k+n-1}
$$

called left action 
- for $i=1, \ldots, k$, morphisms

$$
\bullet_{i}: \mathcal{W}_{k} \otimes \mathcal{C}_{n} \rightarrow \mathcal{W}_{k+n-1}
$$

called right action

satisfying the analogue of the axioms for non-symmetric operads in terms of $\circ_{i}$ operations and a compatibility condition again.

Remark 9.7. It is easy to prove that a weak Ass-Ass-bimodule is the same as a functor $\Delta \rightarrow \mathcal{E}$; that is, a cosimplicial object in $\mathcal{E}$.

We use the notations Bimod and Wbimod for the categories of Ass-Assbimodules and weak Ass-Ass-bimodules respectively.

There is a forgetful functor

$$
v^{*}: \mathrm{NOp}_{*} \rightarrow \text { Bimod. }
$$

The category of pointed bimodules $\operatorname{Bimod}_{*}$ is the category $v^{*} A s s / \operatorname{Bimod}$. We have two forgetful functors

$$
b^{*}: \operatorname{Bimod}_{*} \rightarrow \operatorname{Bimod}
$$

and

$$
w^{*}: \operatorname{Bimod}_{*} \rightarrow \text { Wbimod. }
$$

Proposition 9.8. $\quad$ 1. There are polynomial monads NOp, Bimod and WBimod whose categories of algebras are isomorphic to the categories NOp,Bimod and $\mathrm{WBimod}$ respectively. Moreover, the functors $v^{*}$ and $w^{*}$ are isomorphic to the restriction functors along the maps of polynomial monads:

$$
v: \operatorname{Bimod} \rightarrow N O p_{*} .
$$

and

$$
w: W B i m o d \rightarrow \text { Bimod }_{*} .
$$

2. The monads NOp and Bimod satisfy the conditions of Theorem 8.1, The functors $u^{*}$ and $b^{*}$ are induced by the maps of polynomial monads

$$
u: N O p \rightarrow N O p_{*}
$$

and

$$
b: \operatorname{Bimod}_{\rightarrow} \operatorname{Bimod}_{*} .
$$

Proof. For the description of the monad NOp see Example 2.7

The monads Bimod and WBimod indeed have been described by Turchin in [23] without explicitly saying it. For this reason, and because in Section 10 we will describe a closely related construction, we give only a brief description now. For Bimod, the corresponding polynomial is given by

$$
\mathbb{N} \longleftrightarrow B t r \stackrel{p}{\longrightarrow} B t r \stackrel{t}{\longrightarrow} \mathbb{N} .
$$

Here Btr is the set of isomorphism classes of certain planar trees with black and white vertices (called beads in 23] [p.14]). The restrictions on the class of trees are 23]: 
1. An edge cannot connect two black vertices;

2. If a tree has a white vertex then the path between any leaf or stump (valency one vertex) and the root should pass through one and only one white vertex;

3 . There is exactly one tree without white vertices. This special tree has one black vertex and no leaves (this tree is here to accommodate a special operation described in the Remark 9.4

As Turchin notices, the second condition means that one can draw any such tree $\mathrm{T}$ in a way that all white vertices lie on the same horizontal line.

Remark 9.9. We use a more general type of planar tree here in comparison with 23] [Part 1] because we allow vertices of valencies 1 and 2. This kind of tree is considered in Part 2 of 23 .

The rest of the data for the polynomial monad Bimod is very similar to NOp. The set $B t r^{*}$ is the set $B t r$ with one of the white vertices marked and the middle map, source and target having the same description as for NOp. The multiplication is induced by insertion of a tree to a marked vertex and contraction of all edges connecting black vertices.

The monad WBimod is given by the polynomial

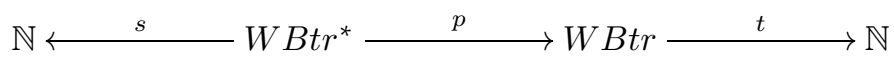

where the class of planar trees $W$ Btr consists of planar trees with black and exactly one white vertex (see [23][p.11]) and the rest of the structure is analogous to the previous.

The polynomial monad $N O p_{*}$ has been described many times in the literature in terms of a $\Sigma$-free $\mathbb{N}$-colored operad of planar trees with white and black vertices (see 4] for a description in terms of trees and lattice paths of complexity 2 as well as for earlier references). The monad $\operatorname{Bimod}_{\star}$ can be given a similar description using the corresponding class of trees. We skip its description here because we will describe a very similar monad in Section 10 .

The maps of polynomial monads $v, w, u$ and $b$ are easy to guess from the restrictions functors $v^{*}, w^{*}, u^{*}$ and $b^{*}$.

\section{First cofinality theorem}

Many constructions of this and subsequent sections are instances of twisted BoardmanVogt tensor product from Section [6. We use some visible special notations for corresponding polynomial monads for a convenience of the reader.

Consider the following polynomial monads:

1. The monad $N O p p_{\bullet \rightarrow \bullet \leftarrow \bullet}$ whose algebras are cospans

$$
\mathcal{A} \rightarrow \mathcal{C} \leftarrow \mathcal{B}
$$

of non-symmetric operads. 
2. The polynomial monad $\operatorname{Bimod}_{\bullet+\bullet}$ whose algebras are triples $(\mathcal{A}, X, \mathcal{B})$ where $\mathcal{A}, \mathcal{B}$ are non-symmetric operads and $X$ is a 1 -pointed $\mathcal{A}-\mathcal{B}$ bimodule.

There is map of polynomial monads

$$
f: \operatorname{Bimod}_{\bullet+\bullet} \rightarrow N_{p_{\bullet} \rightarrow \bullet \leftarrow \bullet}
$$

such that the restriction functor along it forgets operadic structure on $\mathcal{C}$ and remembers only $\mathcal{A}$ - $\mathcal{B}$-bimodule structures induced by two operadic maps as well as the base point in $\mathcal{C}$ given by the unit of the operad $\mathcal{C}$.

Analogously, let Bimod $\bullet \rightarrow \bullet \leftarrow \bullet$ be the polynomial monad whose algebras are cospans

$$
\mathcal{A} \rightarrow \mathcal{C} \leftarrow \mathcal{B}
$$

of Ass-Ass-bimodules.

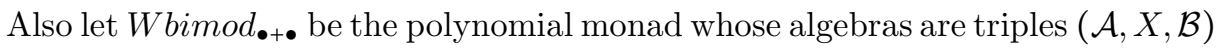
where $\mathcal{A}, \mathcal{B}$ are $A s s-A s s$-bimodules and $X$ is a 0 -pointed $\mathcal{A}$ - $\mathcal{B}$-bimodule. This last object is given by a family of sets $X_{n}, n \geq 0$ together with

1. a point $\star \in X_{0}$

2. left $\mathcal{A}$-action

$$
\mathcal{A}_{p} \times X_{q} \rightarrow X_{p+q}
$$

3. right $\mathcal{B}$-action

$$
X_{p} \times \mathcal{B}_{q} \rightarrow X_{p+q}
$$

4. extension of $X_{n}=X([n])$ to a functor on the subcategory $\Delta_{\text {surj }}$ of $\Delta$ with order-preserving surjections as morphisms.

Both actions are required to be associative and unital with respect to the pairing defined in Remark 9.5 as well as compatible with each other in the usual sense. They also have to be natural with respect to the morphisms in $\Delta_{\text {surj }}$.

Remark 10.1. If in the definition of algebra of $\operatorname{Wbimod}_{\bullet+}$ • the bimodules $\mathcal{A}=\mathcal{B}=1$, are both the terminal Ass-Ass-bimodule then $X$ is a 0 -pointed weak Ass-Assbimodule.

There is a map of polynomial monads

$$
g: \operatorname{Wbimod}_{\bullet+\bullet} \rightarrow \operatorname{Bimod}_{\bullet \rightarrow \bullet \leftarrow \bullet}
$$

such that the restriction functor along it forgets the bimodule structure on $\mathcal{C}$.

Theorem 10.2. The maps

$$
f: \operatorname{Bimod}_{\bullet+\bullet} \rightarrow N O p_{\bullet \rightarrow \bullet \leftarrow \bullet}
$$

and

$$
f: \operatorname{Wbimod}_{\bullet+\bullet} \rightarrow \operatorname{Bimod}_{\bullet \rightarrow \bullet \leftarrow \bullet}
$$

are homotopically cofinal. 
Proof. We have to prove that the classifier $N O p_{\bullet \rightarrow \bullet \leftarrow \bullet}^{\text {Bimod } \bullet \bullet}$ is contractible.

First of all we need explicit descriptions of the polynomial monads $N O p p_{\bullet \rightarrow \bullet} \bullet$ and $\operatorname{Bimod}_{\bullet+\bullet .}$.

The monad $N O p_{\bullet \rightarrow \bullet \leftarrow \bullet}$ is given by the polynomial

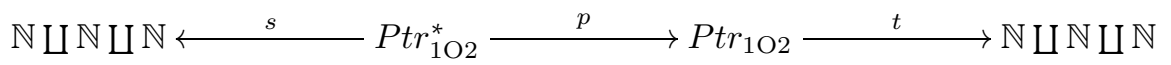

Here, $\operatorname{Ptr}_{1 \mathrm{O} 2}$ is the set of isomorphism classes of planar trees whose vertices can have three colours: white or black of two types 1 or 2 . We also associate one of these colours to each tree (called its target colour). The only condition is that this colour is white type if the tree contains only white vertices or any mixed type vertices. If all vertices of the tree are black of the same type, the target colour can be of the same type as the vertices colour or white. So, for such a tree, we necessary have two copies in the set of operations: one with the corresponding black colour as target and one with the white colour. Also each copy of $\mathbb{N}$ has its own colour (again, white or black, 1 or 2.)

Figure 10.1: Typical tree from $\operatorname{Ptr}_{1 \mathrm{O} 2}$

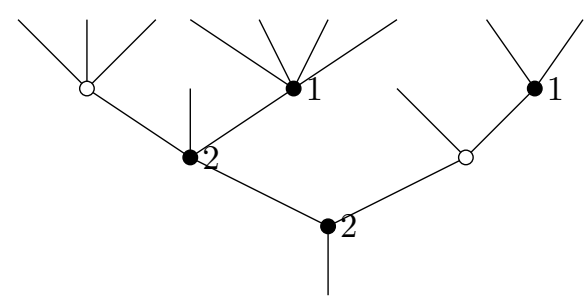

As usual $P t r_{1 \mathrm{O} 2}^{*}$ is the set $\operatorname{Ptr}_{1 \mathrm{O} 2}$ with one vertex marked. The source map produces a natural number of the corresponding colour (white or black 1 or 2) depending on what kind of vertex is marked. The target map returns the number of leaves as before, which is placed to a copy of $\mathbb{N}$ of target colour of the tree.

The polynomial of the monad $\operatorname{Bimod}_{\bullet+\bullet}$ is

$$
\mathbb{N} \amalg \mathbb{N} \amalg \mathbb{N} \longleftarrow s
$$

The set $\operatorname{Ptr}_{1 \mathrm{~B} 2}$ is a subset of $\operatorname{Ptr}_{1 \mathrm{O} 2}$ whose elements subject to the following restrictions :

1. If a tree has all vertices black they must have the same type and the target type of such a tree is also the same;

2. If there is a white vertex in a tree, the path between any leaf or stump and the root should pass through one and only one white vertex (second of Turchin's restrictions), the target type of the tree must be white in this case;

3. Black vertices along a path like the one above have type 1 before the path meets a white vertex and type 2 after the white vertex; 
4. There are exactly three copies of the tree without vertices (a free living edge) each of them having 1 as target but of different types: black 1, black 2 or white.

This last requirement corresponds to the constants in the theory. Each free living edge represents a nullary operation. Operations with black target represent units of each operad, whereas the free livng edge with white target represents the base point in the 1-pointed bimodule.

Figure 10.2: Typical tree from $P \operatorname{tr}_{1 \mathrm{~B} 2}$

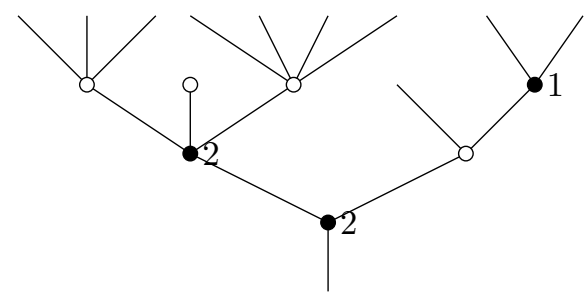

The map $f: \operatorname{Bimod}_{\bullet+\bullet} \rightarrow N O p_{\bullet \rightarrow \bullet} \rightarrow \bullet$ is now obvious. It is the identity on colours and is the natural inclusion on other sets.

We are now ready to describe the classifier $N O p_{\bullet \rightarrow \bullet}^{\text {Bimod }} \bullet \bullet \bullet$

By definition this is a family of categories indexed by natural numbers of three types. If we consider restriction to any of the black colours 1 and 2 the corresponding classifier is isomorphic to the absolute classifier for non-symmetric operads, therefore the category $N O p_{\bullet \rightarrow \bullet \leftarrow \bullet}^{\operatorname{Bimod}_{\bullet} \bullet}\left(n_{b}\right)$, for any $n_{b}$ a 'black' natural number, is contractible. Let us concentrate on the categories $\operatorname{NOp}_{\bullet \rightarrow \bullet \leftarrow \bullet}^{\text {Bimod } \bullet \bullet}\left(n_{w}\right)$ indexed by white natural numbers. So, we fixed one of them. By general machinery of Batanin and Berger [5]:

Proposition 10.3. 1. the objects of this category are trees from $P_{1 \mathrm{O} 2}$ with exactly $n_{w}$ leaves.

2. the morphisms are generated by

(a) contractions to a white vertex of edges where the upper vertices are black of the first type and the lower vertex is white

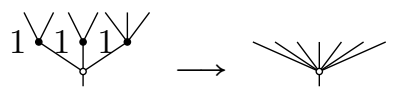

(b) contractions to a white vertex of edges where the upper vertices are white and the lower vertex is black of the second type

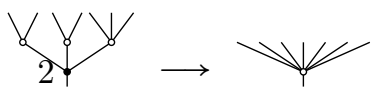


(c) contractions of edges with black vertices of the same type;

(d) insertion of a vertex of valency 2 of any of the types on an edge. For example:

$$
1 \bigvee \rightarrow \bigvee_{i}
$$

3. the relations are generated by the usual bimodules relations, as well as the operadic relations. So, for example, the squares below commute:

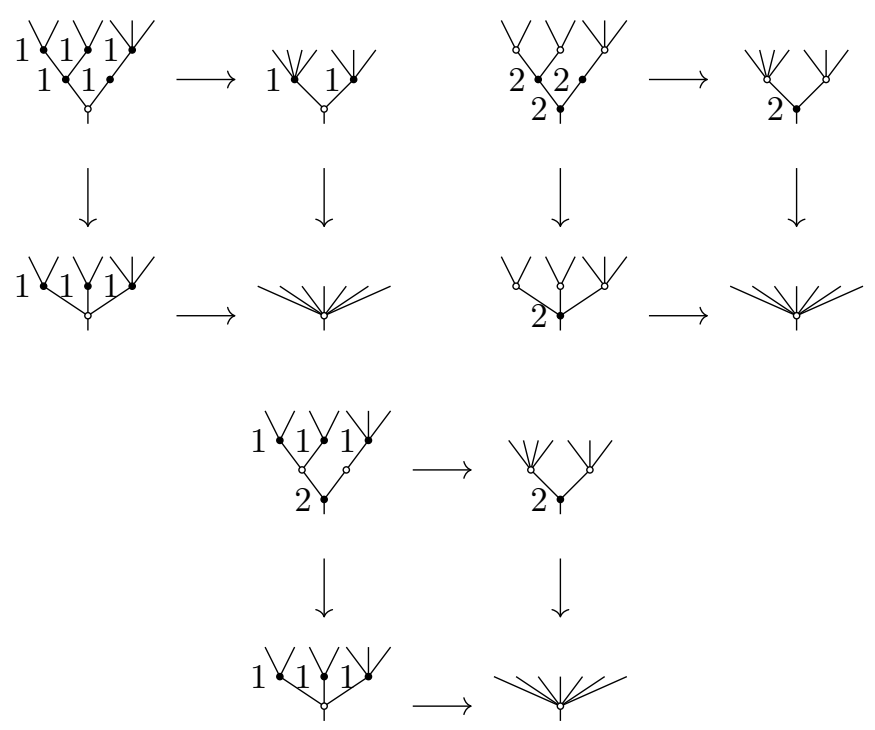

Remark 10.4. The role of the morphism of insertion of a white vertex deserves a separate comment. Combining this morphism with the morphism of bimodule contractions, we obtain two important families of morphisms in the classifier

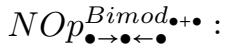

1. Morphisms which replace a black vertex of type 1 by a white vertex:

$$
\text { 1Y } \longrightarrow Y 1 \rightarrow Y
$$

2. Morphisms which replace a black vertex of type 2 by a white vertex

$$
2 Y \rightarrow\{\}_{2} \rightarrow Y
$$

There is one exception to the rule of replacement of a black vertex of type 2, namely, if such a vertex has valency 1 . In this case, however, we have a special operation in the left bimodule (see Remark 9.4) which provides a morphism

$$
2 \uparrow \longrightarrow \text { i }
$$


These morphisms make the classifier $N O p_{\bullet \rightarrow \bullet}^{\text {Bimod }} \bullet_{\bullet} \bullet$ look very similar to the ab-

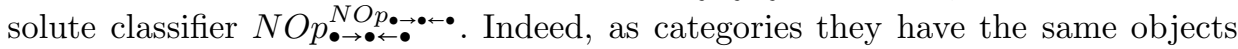
and in both categories there are morphisms of replacing black vertices by white

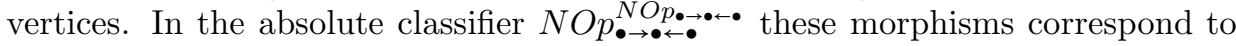
two internal operad morphisms, which is a part of the $N O p_{\bullet} \rightarrow \bullet_{\bullet} \cdot \bullet^{-a l g e b r a ~ s t r u c-~}$

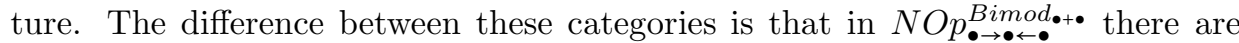
no contractions of edges connecting white vertices, which reflects the fact that we just have an internal bimodule, not an operad.

Remark 10.5. One can develop a very similar theory in the case of reduced operads and bimodules (this is the case of Turchin's paper [23] [Part 1]). Reduced means that operads and bimodules we consider are such that $\mathcal{X}(0)=\varnothing$ and $\mathcal{X}(1)=1$. All $\mathcal{A}$ - $\mathcal{B}$-bimodules are, therefore, canonically 1 -pointed. The corresponding classifiers do not contains vertices of valencies 1 and 2 and, hence, are finite. Indeed they are finite posets. The following is a picture of the category $R N O p_{\bullet \rightarrow \bullet \bullet \bullet}^{R B i m o d} \bullet \bullet \bullet\left(3_{w}\right)$ : 


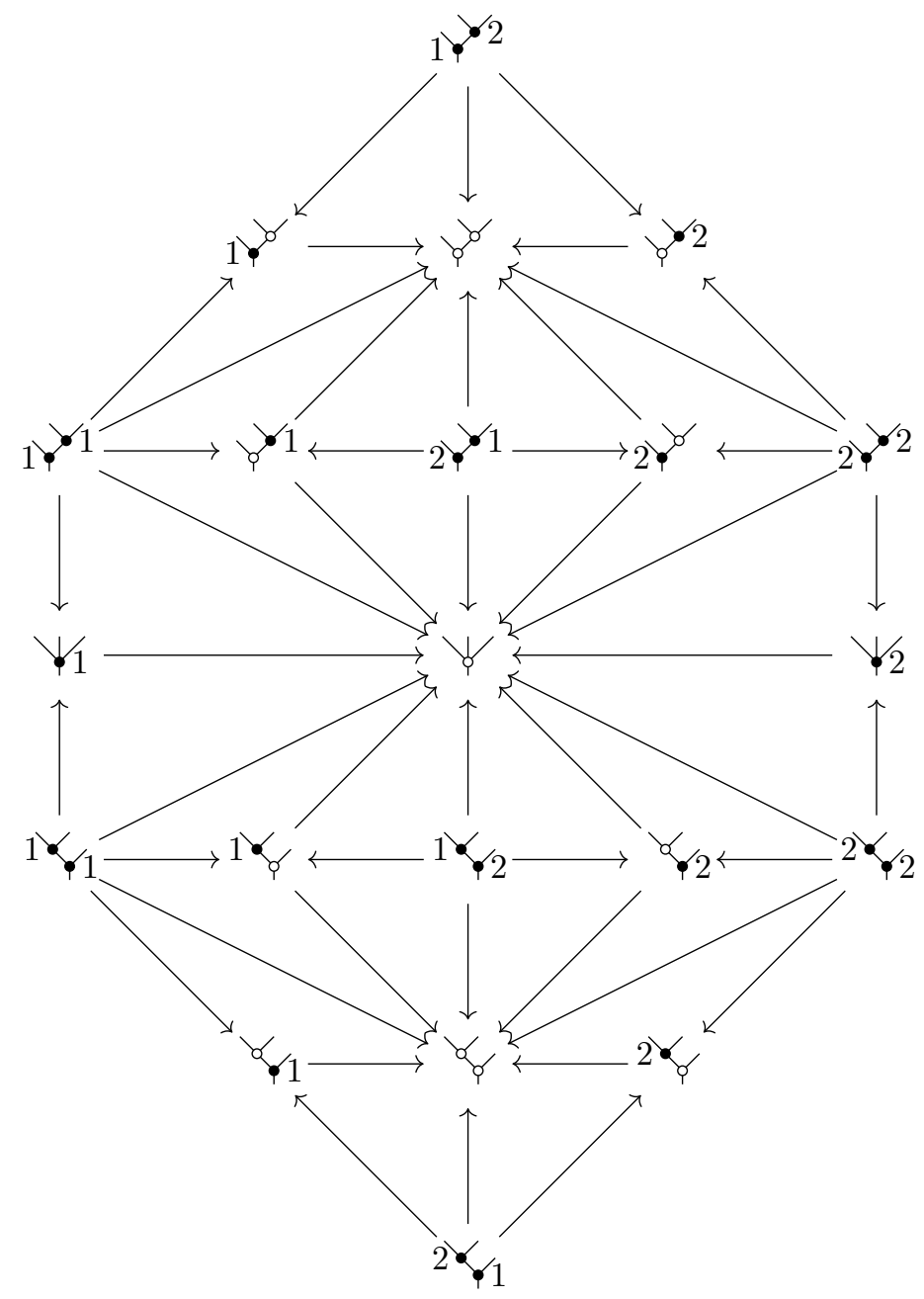

The nerve of this poset is clearly contractible. It is also visible what makes it work. The operadic contractions in this picture are replaced by bimodule contractions. If we are able to invert those morphisms, we get an internal operad structure on corollas with white vertices together with two operadic maps from two internal operads formed by black corollas. This is the conceptual main point of our theorem.

We continue with a formal proof of contractibility of the classifier $N O p p_{\bullet \rightarrow \bullet}^{\text {Bimod } \bullet \bullet \bullet}$.

There is a map of polynomial monads

$$
u: N O p \bullet \rightarrow \bullet \bullet \bullet N O p
$$

constructed as follows. On colours

$$
\mathbb{N} \coprod \mathbb{N} \coprod \mathbb{N} \rightarrow \mathbb{N}
$$


it is an identity on each summand. On operations it forgets about all colours (vertices colours as well as target colours) of trees from $\mathrm{Ptr}_{1 \mathrm{O} 2}$. In other words it only remembers the shape of the tree. This is a cartesian map and the restriction functor along this map applied to an operad $\mathcal{O}$ returns a cospan $\mathcal{O} \stackrel{i d}{\rightarrow} \mathcal{O} \stackrel{i d}{\leftarrow} \mathcal{O}$. We then have the following commutative square of cartesian maps of polynomial monads:

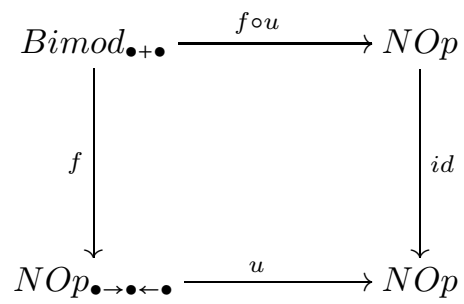

By Proposition 4.7, we then have a map of classifiers

$$
F: N O p_{\bullet \rightarrow \bullet \leftarrow \bullet}^{\text {Bimod•*• }} \rightarrow u^{*}\left(N O p^{N O p}\right) .
$$

We are going to prove that the nerve of this map is a weak equivalence which, of course, will imply the contractibility of $N O p p_{\bullet \rightarrow \bullet \leftarrow \bullet}^{\text {Bimod•• }}$. The map (23) on objects has the same effect as (21) on operations. It also maps contractions to contractions while any morphism which comes from replacement of black vertices by white vertex is mapped to the identity.

We fix a particular $n \in \mathbb{N}$ and consider the restriction of the map of the classifiers (23) to the component indexed by $n_{w}$ (it is clear that on components with black colours the map (23) is an isomorphism). To make our notation less heavy we give names to two of our categories as follows:

$$
\operatorname{NOp}_{\bullet \rightarrow \bullet \leftarrow \bullet}^{\operatorname{Bimod} \bullet \bullet}\left(n_{w}\right)=\mathbb{C}
$$

and

$$
u^{*}\left(N O p^{N O p}\right)\left(f\left(n_{w}\right)\right)=\mathbb{D} .
$$

The restriction of (23) on this components will simply be denoted by $F$.

For any $d \in \mathbb{D}$, we write $F_{d}$ for the (strict) fiber of $F$ over $d$; that is, the full subcategory of objects $c \in \mathbb{C}$ such that $F(c)=d$.

Definition $10.6([8])$. A functor $F: \mathbb{C} \rightarrow \mathbb{D}$ is smooth if, for all $d \in \mathbb{D}$, the canonical functor

$$
F_{d} \rightarrow d / F
$$

induces a weak equivalence of nerves.

Dually a functor $F: \mathbb{C} \rightarrow \mathbb{D}$ is proper if, for all $d \in \mathbb{D}$, the canonical functor

$$
F_{d} \rightarrow F / d
$$

induces a weak equivalence of nerves.

We have the following lemma [8, Proposition 5.3.4] : 
Lemma 10.7. A functor $F: \mathbb{C} \rightarrow \mathbb{D}$ is smooth if and only if for all maps $f_{1}: d_{0} \rightarrow$ $d_{1}$ in $\mathbb{D}$ and all objects $c_{1}$ in $F_{d_{1}}$, the nerve of the 'lifting' category $\mathbb{C}\left(c_{1}, f_{1}\right)$ of $f_{1}$ over $c_{1}$, whose objects are arrows $f: c \rightarrow c_{1}$ such that $F(f)=f_{1}$ and arrows are commutative triangles :

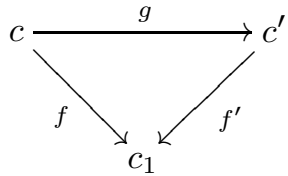

with $g$ a morphism in $F_{d_{0}}$, is contractible.

There is a dual characterisation for proper functors.

We call any tree in $\mathbb{C}$ which has only one vertex a corolla. We call the unique vertex connected to the root vertex. The root path from a vertex is the path from this vertex to the root vertex. Using a description of the category $\mathbb{C}$ from Proposition 10.3, we have the following lemma:

Lemma 10.8. [Characterisation of trees that can be contracted to a corolla] Let $c \in \mathbb{C}$ be a corolla. There is a morphism from a tree a to $c$ in $\mathbb{C}$ if and only if the tree a satisfies the following property:

* the root path from any vertex consists of a sequence of black vertices of type 1 followed by at most one white vertex followed by a sequence of black vertices of type 2 .

Figure 10.3: Tree satisfying the property *

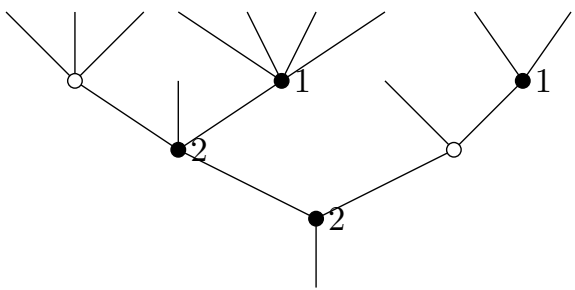

Proof. Assume that $a$ satisfies the property $*$. We use induction on the maximal length of a root path in the tree $a$. If $a$ is a corolla, then the statement is trivial. If $a$ is not a corolla, one can consider $a$ to be a forest of branches joined at the root vertex. All the branches satisfy the property $*$ and have maximal path root length strictly less than $a$. By induction, they can therefore be contracted to a corolla. If the root vertex is black of type 2 , the remaining tree can always be contracted. If not, then all the vertices above the root vertex in $a$ are black of type 1, and the remaining tree can also be contracted.

For the other direction of the equivalence, assume that $a$ does not satisfy the property $*$. Notice that if a tree $a$ does not satisfy the property $*$ and $a \rightarrow b$ is a generating morphism, then $b$ does not satisfy the property $*$. Yet the corolla satisfies the property $*$, which is a contradiction. 
Lemma 10.9. The functor $F: \mathbb{C} \rightarrow \mathbb{D}$ is smooth.

Proof. Let $f_{1}: d_{0} \rightarrow d_{1}$ in $\mathbb{D}$ and $c_{1}$ in $F_{d_{1}}$. According to the Lemma 10.7, we have to prove that the nerve of the category $\mathbb{C}\left(c_{1}, f_{1}\right)$ is contractible.

Observe that $\mathbb{C}\left(c_{1}, f_{1}\right)$ is isomorphic to the full subcategory of $\mathbb{C}$ consisting of trees with the same shape as $d_{0}$ which can be contracted to $c_{1}$. Moreover, $\mathbb{C}\left(c_{1}, f_{1}\right)$ is also isomorphic to a product of categories $\mathbb{C}\left(c_{1}^{v}, f_{1}^{v}\right)$ where

- $v$ runs over the vertices of $d_{1}$

- $d_{1}^{v}$ is the corolla whose set of leaves is equal to the set of incoming edges of $v$

- $d_{0}^{v}$ is the subtree of $d_{0}$ containing all the vertices that are sent to $v$ through $f_{1}$

- $f_{1}^{v}: d_{0}^{v} \rightarrow d_{1}^{v}$ is the contraction to the corolla

- $c_{1}^{v}$ is the corolla with the same color as the vertex $v$ of $c_{1}$

If the unique vertex of $c_{1}^{v}$ is black, then $\mathbb{C}\left(c_{1}^{v}, f_{1}^{v}\right)$ is the terminal category and its nerve is contractible. We can therefore assume that $c_{1}^{v}$ is a corolla with a white vertex.

In conclusion, all we have to prove is the contractibility of the nerve of $\mathbb{C}_{d}$, which is defined as the subcategory of trees in $\mathbb{C}$ with the same shape as $d \in \mathbb{D}$ and that can be contracted to a corolla with a white vertex.

Let us introduce the following notations :

- We write $\mathbb{C}^{1}$ for the full subcategory of $\mathbb{C}_{d}$ consisting of trees whose all non root vertices are black of type 1 .

- We write $\mathbb{C}_{2}$ for the full subcategory of $\mathbb{C}_{d}$ consisting of trees whose root vertex is black of type 2 . 
Figure 10.4: The subcategories $\mathbb{C}^{1}$ and $\mathbb{C}_{2}$

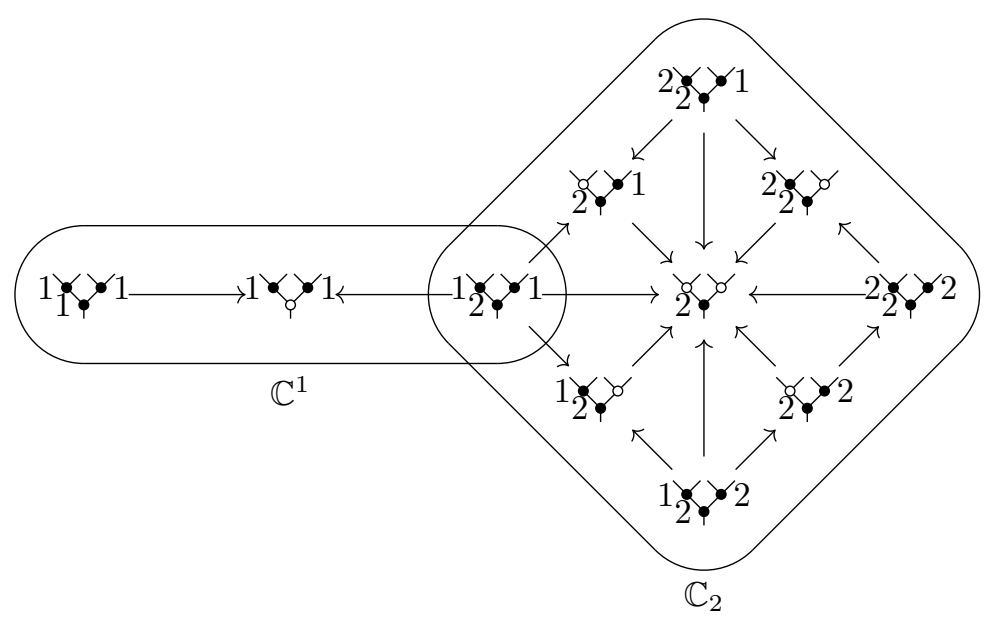

Using lemma 10.8, we deduce that $\mathbb{C}_{d}=\mathbb{C}^{1} \cup \mathbb{C}_{2}$.

The subcategory $\mathbb{C}^{1}$ contains only 3 objects, and one of them is terminal for this subcategory, namely, the tree where the root vertex is white and all other vertices are black of type 1 .

The subcategory $\mathbb{C}_{2}$ is isomorphic to the product of the subcategories $\mathbb{C}_{d_{1}}, \ldots, \mathbb{C}_{d_{k}}$, where $d_{1}, \ldots, d_{k}$ are the subtrees coming up from the root vertex of $d$. Indeed, an object $c \in \mathbb{C}_{2}$ can be associated to a sequence of objects $c_{1}, \ldots, c_{k} \in \mathbb{C}_{d_{1}}, \ldots, \mathbb{C}_{d_{k}}$ by taking the subtrees coming up from the root vertex of $d$. This association is obviously a bijection thanks to the characterization of lemma 10.8. By induction, the nerves of $\mathbb{C}_{d_{1}}, \ldots, \mathbb{C}_{d_{k}}$ are contractible. Therefore, the nerve of $\mathbb{C}_{2}$ is also contractible.

Finally, the intersection $\mathbb{C}^{1} \cap \mathbb{C}_{2}$ contains only one object, namely the tree where the root vertex is black of type 2 and all the other vertices are black of type 1.

To prove the first part of Theorem 10.2 it remains to show that the fiber $F_{d}$ is contractible for any $d \in \mathbb{D}$. This was already observed in the proof of Lemma 10.9.

For the proof of the second part of Theorem 10.2, we use a very similar scheme. Because of this we give only a brief outline of the proof, mostly focussing on the differences between the two cases.

Both polynomial monads Bimod $\operatorname{Bi\bullet }_{\bullet} \leftarrow$ and $\operatorname{Wbimod}_{\bullet+\bullet}$ have $\mathbb{N} \amalg \mathbb{N} \amalg \mathbb{N}$ as their colours and both involve specifically decorated planar trees.

For Bimod. $\rightarrow \bullet \leftarrow \bullet$, we use trees which now have four different types of vertices: white, black of type 1 or 2 and black vertices without any type. An edge cannot connect two black vertices without type, and the path between any leaf or stump 
(valency one vertex) and the root should pass through one and only one vertex which is white or black of type 1 or 2 .

Figure 10.5: A typical tree for $\operatorname{Bimod}_{\bullet \rightarrow \bullet \leftarrow \bullet}$

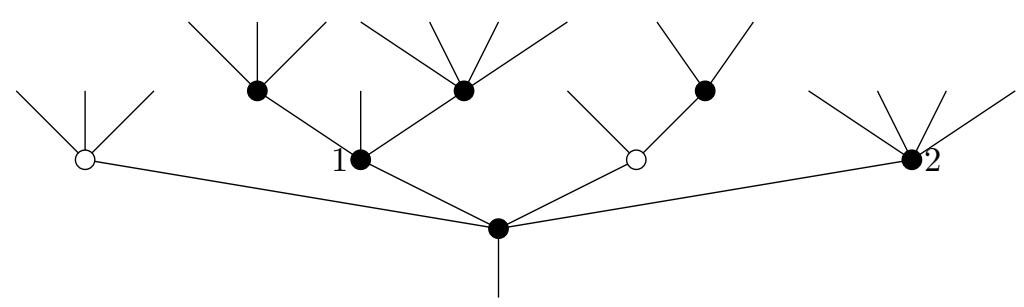

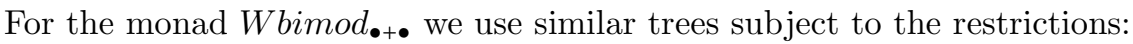

1. A tree may have at most one white vertex;

2. Black vertices of type 2 are on the left of the white vertex and black vertices of type 1 are on the right of the white vertex;

3. If a tree has all vertices black, the typed vertices must have the same type;

4. There are exactly three copies of the tree without vertices (a free living edge) each of them having 0 as target but of different types: black 1, black 2 or white.

\section{Figure 10.6: A typical tree for Wbimod $_{\bullet+\bullet}$}

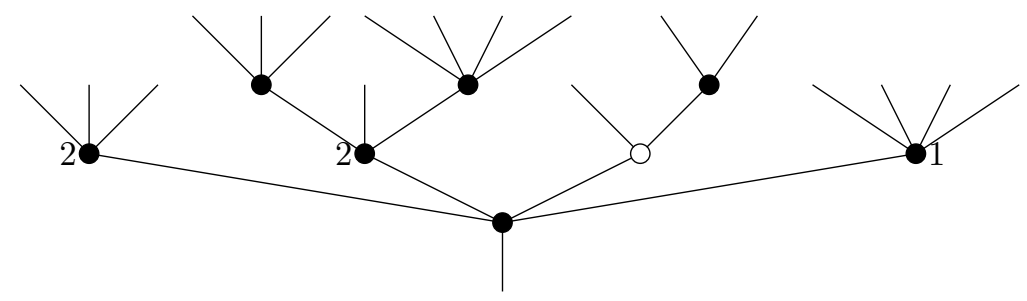

The map $f:$ Wbimod $_{\bullet+\bullet} \rightarrow \operatorname{Bimod}_{\bullet \rightarrow \bullet \leftarrow \bullet}$ is now obvious. It is the identity on colours and is the natural inclusion on other sets.

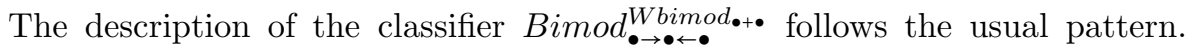

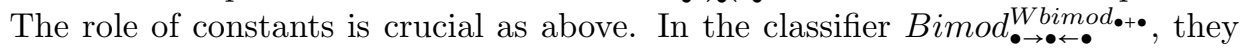
generate the morphisms of insertion of a stump on one side of a tree. In particular, we have the following morphisms of replacement:

1. Morphism replacing a black vertex of type 1 by a white vertex:

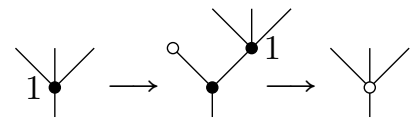


2. Morphism replacing a black vertex of type 2 by a white vertex

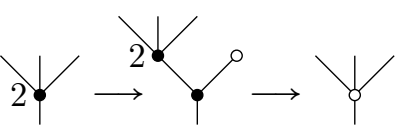

These two morphism classes are enough to proceed with a proof of contractibility

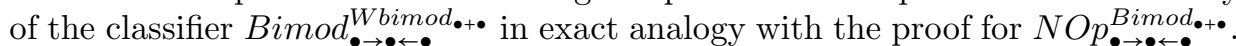

Remark 10.10. One can again consider a reduced version of the theory which in this context means that bimodules and weak bimodules we consider have unique operations in degrees 0 and 1 as in Turchin's paper 23] [Part 1]. In particular, such weak bimodules are 0-pointed automatically. The corresponding classifiers do not contains vertices of valency 1 and 2 and, hence, are finite posets. The following is a picture of the category $\operatorname{RBimod}_{\bullet \rightarrow \bullet \leftarrow \bullet}^{R W \operatorname{Wimod}_{\bullet \bullet \bullet}}\left(2_{w}\right)$ :

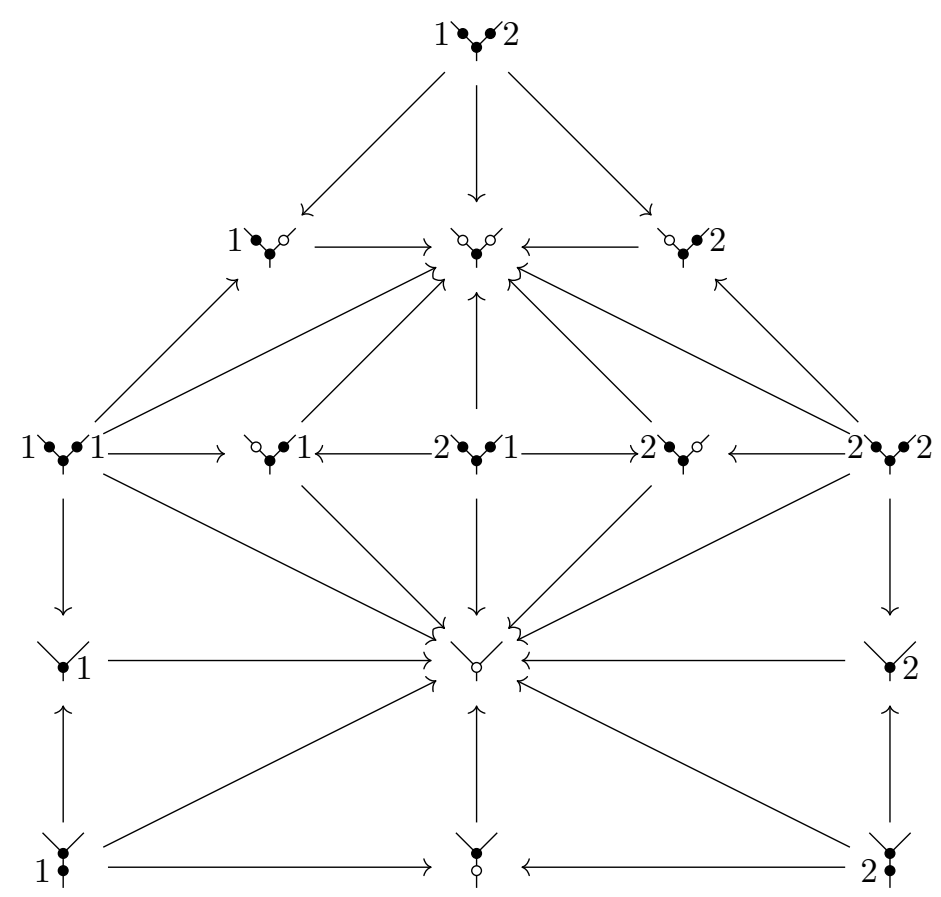

The nerve of this poset is clearly contractible.

\section{Second cofinality theorem}

Let Bimod ${ }_{+}$be the category of 1-pointed Ass-bimodules. Analogously, let WBimod ${ }_{+}$ be the category of 0-pointed weak Ass-bimodules. These categories are categories 
of algebras of polynomial monads $\mathrm{Bimod}_{+}$and $\mathrm{WBimod}_{+}$respectively obtained by the pushouts described in the Theorem 8.3 .

Theorem 11.1. There are homotopically cofinal maps of polynomial monads

$$
f: \operatorname{Bimod}_{+} \rightarrow N O p_{* *}
$$

and

$$
g: \text { Bimod }_{+} \rightarrow \operatorname{Bimod}_{* *}
$$

Proof. As before we give the details of the proof of only the first part of the theorem. The proof of the part concerning bimodules versus weak bimodules is very similar and we leave it as an exercise.

Firstly we have to describe the monads $N O p_{* *}$ and $\operatorname{Bimod}_{+}$explicitly. The first monad is represented by a polynomial

$$
\mathbb{N} \longleftrightarrow{ }^{s}-P t r_{* \mathrm{O} *}^{*} \longrightarrow p t r_{* \mathrm{O} *} \longrightarrow \frac{t}{\longrightarrow} \mathbb{N} .
$$

Here, $P t r_{* \mathrm{O} *}$ is the subset of $P t r_{1 \mathrm{O} 2}$ consisting of trees with a condition that no edge can connect two black vertices of the same type. Similarly, $P t r_{* \mathrm{O} *}$ is the subset of $P t r_{102}^{*}$ with one white vertex marked (so we do not allow insertion to black vertices). The rest of the description of the monad is very similar to the $\operatorname{monad} N O p_{\bullet} \rightarrow \bullet \leftarrow \bullet \cdot$

Analogously, the monad Bimod + is represented by a polynomial

$$
\mathbb{N} \longleftrightarrow P^{s} r_{* \mathrm{~B} *}^{*} P t r_{* \mathrm{~B} *} \stackrel{p}{\longrightarrow} \mathbb{N}
$$

where $P t r_{* \mathrm{~B} *} \subset P t r_{1 \mathrm{~B} 2}$ whose elements are subject to the condition as above. The rest of the structure is also similar to the monad $\operatorname{Bimod}_{\bullet+\bullet}$. It is also clear how to construct a map

$$
f: \mathrm{Bimod}_{+} \rightarrow N O p_{* *} .
$$

We are going to prove that this map of polynomial monads is homotopically cofinal.

We will need another map of polynomial monads intermediate between (19) and (24). Namely, let $N O p_{\circ \rightarrow \bullet \leftarrow \circ}$ be the monad whose algebras are cospans of non-symmetric operads

$$
\mathcal{A} \rightarrow \mathcal{C} \leftarrow \mathcal{B}
$$

where $\mathcal{A}$ and $\mathcal{B}$ are such that $\mathcal{A}(1) \cong \mathcal{B}(1) \cong 1$.

Remark 11.2. We will call such non-symmetric operads semireduced because they do not have nontrivial unary operations but may have nontrivial operations of arity 0 .

Explicitly, such a monad given by a polynomial similar to (20) but corresponding trees are semireduced; that is, they do not contain black vertices of valency 2 . Also, the colours for black types are now $\mathbb{N} \backslash\{1\}$.

Also, let Bimod o+o $_{\circ}$ be the monad whose algebras are given by triples of two semireduced operads and a 1-pointed bimodule over them. We have a map of the monads

$$
f: \text { Bimod }_{\circ+\circ} \rightarrow N O p_{\circ \rightarrow \bullet \leftarrow \circ} .
$$


All these monads are included in the following commutative diagram of cartesian maps:

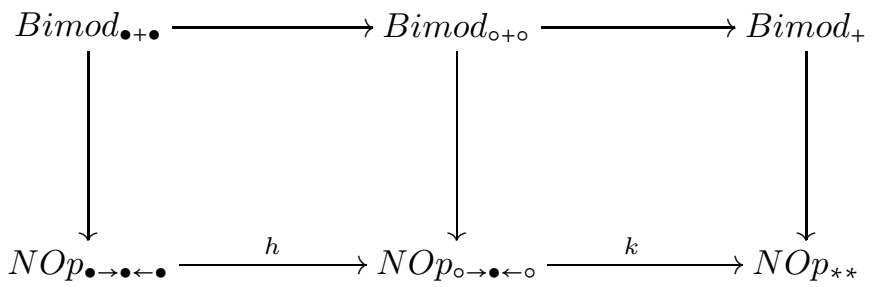

where the horizontal map $h$ acts on colours as follows: on each $\mathbb{N}_{b_{i}}, i=1,2$ it is defined by $h(n)=n, n \neq 1$ and $h(1)=0$. On $\mathbb{N}_{w}$ it acts identically. On a tree it erases all valency 2 black points. The horizontal map $k$ on colours sends each element from black summand $\mathbb{N}_{b_{i}}$ to 0 and it is an identity on $\mathbb{N}_{w}$. On operations it sends a tree to the maximal possible contraction of this tree with respect to operadic contractions of black vertices. In other words, it contracts each edge which connects two black vertices of the same type to a black vertex of this type. The top horizontal maps act similarly.

The square (25) induces the following maps of classifiers

$$
\begin{gathered}
G: N O p_{\bullet \rightarrow \bullet \leftarrow \bullet}^{\text {Bimod }_{\bullet} \bullet} \rightarrow h^{*}\left(N_{0} p_{\circ \rightarrow \bullet \leftarrow \circ}^{\text {Bimod }_{\circ+\circ}}\right) \\
E: N O p_{\circ \rightarrow \bullet \leftarrow \circ}^{\text {Bimod }_{\circ+\circ}} \rightarrow k^{*}\left(N O p_{* *}^{\text {Bimod }_{+}}\right)
\end{gathered}
$$

Lemma 11.3. The underlying functor of $G$ has a left adjoint $K$.

Proof. Let $n$ be one of the colours of the monad $N O p_{\bullet \rightarrow \bullet \leftarrow \bullet}$. We will denote the underlying functor of $G$ restricted to $n$ by the same letter $G$ to simplify the language. Then $G$ acts on objects of $N O p$ Bimod Bim $_{\bullet}$ by erasing valency 2 black points. We define $K$ on objects to be an inclusion of semireduced trees to the set of all trees. We claim that this inclusion can be extended to a functor.

Indeed, the set of generators for morphisms in the classifier $N O p_{\circ \rightarrow \bullet \leftarrow \circ}^{B i m o d_{0+0}}$ is the same as in Proposition 10.3 except that in (d) we do not allow the insertion of black points on an edge. The relations are also the same as in Proposition 10.3 , To define $K$ on morphisms is to define it on generators and we simply can do it by mapping a generator to the corresponding generator. Since relations are the same we get a functor.

Finally, the unit of the adjunction is the identity and the counit is a morphism $K G(a) \rightarrow a$ which inserts all black points back to $a$ after $G$ erases them. It is trivial to check that unit and counit satisfy the two triangle relations.

Lemma 11.4. The underlying functor of $E$ is proper and has contractible fibres.

Proof. To see this we first want to prove that the underlying categories of $\mathbb{C}=$ $N O p_{\mathrm{o} \rightarrow \bullet \leftarrow 0}^{\text {Bimod }_{0} \text { o }}$ and $\mathbb{D}=N O p_{* *}^{\text {Bimod }_{+}}$are posets.

For an object $a \in \mathbb{C}$, let us denote by $(\mathbb{C}, a)$ the full subcategory of $\mathbb{C}$ spanned by the objects $b$ for which there exists a morphism $b \rightarrow a$. Observe that by construction of classifiers the category $(\mathbb{C}, a)$ is a product of categories $\left(\mathbb{C}, a^{v}\right)$ where $v$ runs 
over the vertices of $a$ and $a^{v}$ is the corolla determined by $v$ (that is, a corolla whose unique vertex has the same colour as $v$ and whose set of leaves is equal to the set of incoming edges of $v$ ). Hence, it is enough to prove that each $\left(\mathbb{C}, a^{v}\right)$ is a poset. Moreover, it is enough to prove that $a^{v}$ is the terminal object in this category.

If $v$ is a black vertex of a type $i=1,2$, it is clear that $\left(\mathbb{C} a^{v}\right)$ is isomorphic to the category of trees and their contractions whose all vertices are black of the same type $i$. This is a poset with the terminal object $a^{v}$ as easily follows from axioms of semireduced non-symmetric operad.

If $v$ is a white vertex, we can use Lemma 10.8 to describe the objects of $\left(\mathbb{C}, a^{v}\right)$. All morphisms are operadic contractions between black vertices and bimodule contractions for white and black vertices. The fact that $a^{v}$ is terminal here follows from the bimodule relations by a standard combinatorial technique involving the diamond lemma and induction on the number of black vertices.

A similar kind of proof works for $\mathbb{D}$.

The fiber $E_{d}$ of the functor $E$ over any tree $d \in \mathbb{D}$ contains $d$ as well. Moreover, it is very obvious that $d$ is the terminal object of this fiber. So, given $f: d_{0} \rightarrow d_{1}$ in $\mathbb{C}$ and $c \in E_{d_{0}}$, an object of the lifting category of $f$ under $c_{0}$ is just any morphism $g: c_{0} \rightarrow c_{1}$ where $c_{1} \in E_{d_{1}}$. Since $d_{1}$ is the terminal object in $E_{d_{1}}$, we have a unique morphism from $u: c_{1} \rightarrow d_{1}$ and hence, a morphism in the lifting category from $g \rightarrow g \cdot u$. Since we are in a poset, the morphism $g \cdot u$ does not depend on $g$. Hence, it is the terminal object of the lifting category. This completes the proof.

Remark 11.5. To illustrate the idea of the proof above one can consider a reduced

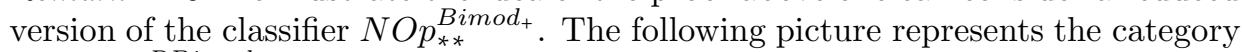
$d^{*} R N O p_{* *}^{R \text { Rimod }_{+}}\left(3_{w}\right)$ which is the target of the functor $G\left(3_{w}\right)$. The source of this functor is represented by the category shown at Remark 10.5 . 


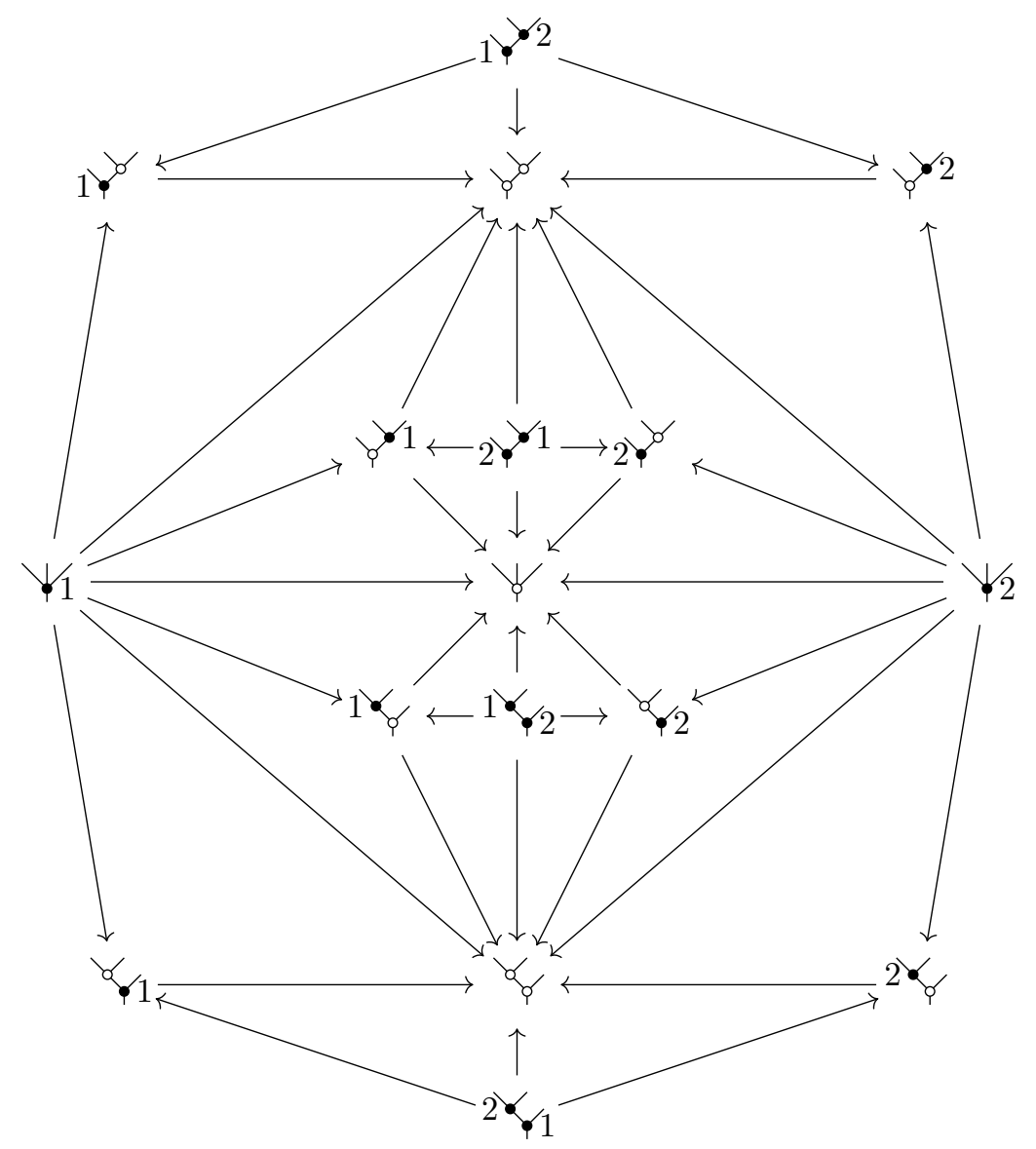

The functor $G$ simply 'shrinks' certain faces of the polytope from 10.5 This corresponds exactly to the contraction discribed by Turchin in [23] [p. 34].

We can finish the proof of Theorem 11.1 by observing that $N(G)$ is a weak equivalence (since $G$ is a right adjoint) and $N(E)$ is a weak equivalence (since it is proper with contractible fiber).

\section{Dwyer-Hess-Turchin's delooping theorems}

Our goal is to prove the following theorem first established independently in [11] and 23 .

Theorem 12.1. For any simplicial multiplicative operad $\mathcal{O}$, there exists a fibration sequence of simplicial sets

$$
\Omega M a p_{\mathrm{NOp}}\left(u^{*} A s s, u^{*} \mathcal{O}\right) \rightarrow \operatorname{Map}_{\text {Bimod }}\left(v^{*} A s s, v^{*} \mathcal{O}\right) \rightarrow f i b\left(\mathcal{O}_{1}\right) .
$$


For any simplicial pointed bimodule $\mathcal{B}$, there exists a fibration sequence of simplicial sets

$$
\Omega M a p_{\text {Bimod }}\left(v^{*} A s s, b^{*} \mathcal{B}\right) \rightarrow M a p_{\text {WBimod }}\left(w^{*} v^{*} A s s, w^{*} b^{*} \mathcal{B}\right) \rightarrow f i b\left(\mathcal{B}_{0}\right) .
$$

This theorem implies the explicit double delooping formula of Dwyer-HessTurchin:

Corollary 12.2. Let a simplicial multiplicative operad $\mathcal{O}$ be such that $\mathcal{O}_{0}$ and $\mathcal{O}_{1}$ are contractible. Then there is a weak equivalence of simplicial spaces

$$
\operatorname{holim}_{\Delta}\left(\mathcal{O}^{*}\right) \sim \Omega^{2} \operatorname{Map}_{\mathrm{NOp}}\left(u^{*} A s s, u^{*} \mathcal{O}\right),
$$

where $\mathcal{O}^{*}$ is the cosimplicial object associated to multiplicative operad $\mathcal{O}$.

Proof of Theorem [12.1. Since $f: \operatorname{Bimod}_{+} \rightarrow N O p_{* *}$ is homotopically cofinal the result will follow from Theorems 8.1 and 8.3 if we know that the squares 26 and 27 are homotopically cofinal. This is the content of two lemmas below.

The proof for the second fibration sequence is similar.

Lemma 12.3. The square

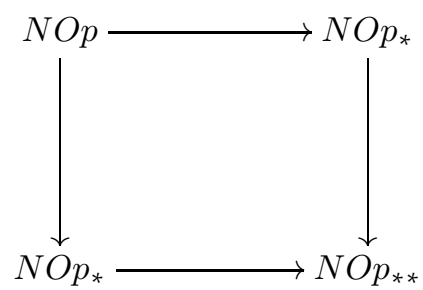

is homotopically cofinal.

Proof. According to the Theorem 6.12, we have to prove that the nerve of $N O p_{* *}^{\phi F}$ is contractible, where $F$ is the presheaf of polynomial monads given by the span

$$
N O p_{*} \leftarrow N O p \rightarrow N O p_{*} .
$$

Using Proposition 7.4 and Theorem 6.12, the nerve of $N O p^{\oint N O p}$ is contractible, where $N O p$ is the constant presheaf

$$
N O p \leftarrow N O p \rightarrow N O p .
$$

It will be enough to prove that $N O p_{* *}^{\oint F}$ and $N O p^{\oint N O p}$ as categories over $\mathbb{N}$ are connected by a sequence of adjoint functors.

According to the Remark 7.5 the objects of the category $N O p_{* *}^{\phi F}$ are given by trees with two types of black vertices 1,2 and three types of white vertices $0,1,2$, with the condition that no edge can connect two black vertices of the same type.

The morphisms are generated by: 
- transformations of a black vertex to a white vertex of the same type or transformations of a white vertex of type 0 to a white vertex of type 1 or to a white vertex of type 2 :

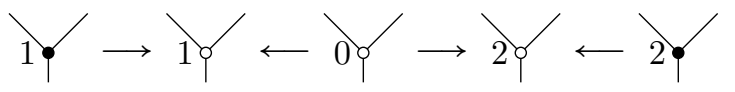

- operadic contractions of edges connecting white vertices of the same type

The category $N O p^{\oint N O p}$ is the full subcategory of $N O p_{* *}^{\oint F}$ containing trees with only white vertices.

We define alt $\left(N O p^{\oint N O p}\right)$ as the full subcategory of $N O p^{\oint N O p}$ containing trees with the condition that no edge can connect two white vertices of type 1 or two white vertices of type 2 . Similarly, we define alt $\left(N O p_{* *}^{\oint F}\right)$.

Then the inclusion

$$
\operatorname{alt}\left(N O p^{\oint N O p}\right) \hookrightarrow N O p^{\oint N O p}
$$

has a left adjoint that sends a tree to the same tree but where the edges connecting two whites vertices of type 1 or two white vertices of type 2 .

Similarly, there is an adjunction between alt $\left(N O p_{* *}^{\oint F}\right)$ and $N O p_{* *}^{\oint F}$.

Finally, the inclusion

$$
\operatorname{alt}\left(N O p^{\oint N O p}\right) \hookrightarrow \operatorname{alt}\left(N O p_{* *}^{\oint F}\right)
$$

has a left adjoint that sends a tree to the same tree but where the black vertices have been turned into whites vertices of the same type.

In summary, we have a sequence of adjunctions

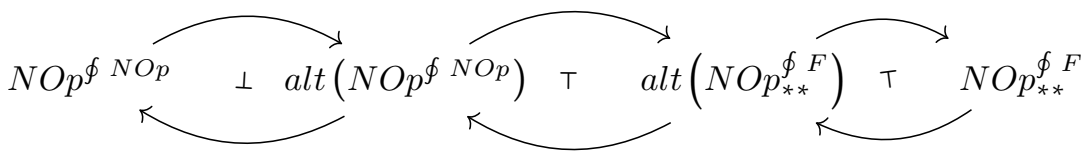

which concludes the proof.

Lemma 12.4. The square

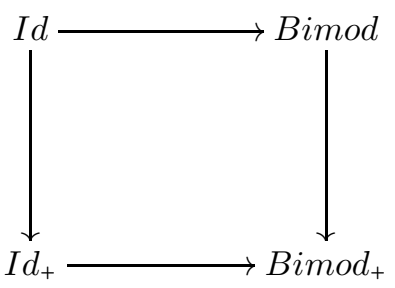

is homotopically cofinal. 
Proof. This time we have to prove that the nerve of $\operatorname{Bimod}_{+}^{\oint} F$ is contractible, where $F$ is the presheaf of polynomial monads given by the span

$$
I d_{+} \leftarrow I d \rightarrow \text { Bimod }
$$

As in the previous Lemma we will exhibit a string of adjunctions connecting the category $\operatorname{Bimod}_{+}^{\oint F}$ (over $\mathbb{N}$ ) and a subcategory of $\operatorname{Bimod}_{+}^{\oint F}$ whose nerve is contractible.

The objects in $\operatorname{Bimod}_{+}^{\oint F}$ are trees with two types of black vertices 1,2 and three types of white vertices $0,1,2$. The conditions are

- the white vertices of type 0 and 1 can occur only if the vertex has valency 2 , that is only one incoming edge

- there can be no edge between two black vertices of the same type

- any path from a leaf and the root meets possibly a black vertex of type 1 , then possibly a white vertex of any type, then possibly a black vertex of type 2

Remark that these conditions imply that if a vertex is black of type 2 , it can only be the root vertex.

The morphisms in Bimod $_{+}^{\oint F}$ are generated by:

- transformations of a white vertex of type 0 to a white vertex of type 1 or to a white vertex of type 2

$$
1\} \longleftarrow 0 \nmid \longrightarrow 2\}
$$

- addition of an unary white vertex of type 1 above a black vertex of type 2 or below a black vertex of type 1, as long as the tree obtained is still in the set of objects, for example

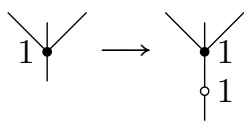

- bimodules operations when there are black vertices of type 1 above white vertices of type 2 or when there are white vertices of type 2 above a black vertex of type 2

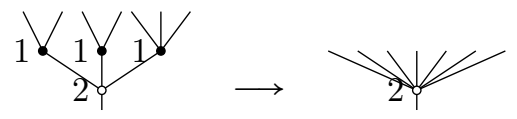

or

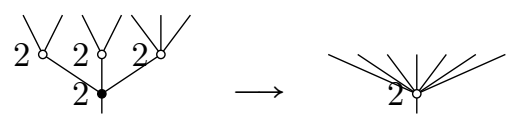


First, let us define $W_{012}$ as the full subcategory of $\operatorname{Bimod}_{+}^{\phi} F$ containing the trees for which the path from any leaf to the root vertex contains exactly one white vertex.

The inclusion

$$
W_{012} \hookrightarrow \text { Bimod }_{+}^{\oint F}
$$

has a left adjoint given which sends a tree to same tree but where we add a unary white vertex of type 1 on all the paths from a leaf to the root vertex which do not contain a white vertex.

Now, we define $W_{2}$ as the full subcategory of $W_{012}$ containing trees where white vertices are only of type 2 . It is obvious that $W_{2}$ is isomorphic to Bimod ${ }^{\text {Bimod }}$.

We then have a sequence of adjunctions

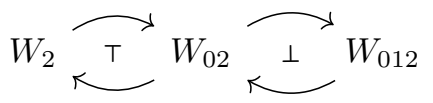

where $W_{02}$ is the full subcategory of $W_{012}$ containing trees where white vertices are only of type 0 or 2 and the functors $W_{02} \rightarrow W_{2}$ and $W_{012} \rightarrow W_{02}$ turn white vertices of type 0 to white vertices of type 2 and white vertices of type 1 to white vertices of type 0 respectively.

This concludes the proof.

Acknowledgements. We wish to express our gratitude to B. Fresse, A.Lazarev, R. Street, S. Lack, R. Garner, B. Shoikhet, V. Turchin, for many useful discussions.

The first author also gratefully acknowledges the financial support of Max Planck Institut für Mathematik.

\section{Bibliography.}

[1] Batanin M. A., An operadic proof of Baez-Dolan stabilization hypothesis, arXiv:1511.09130,

[2] Batanin M. A., The symmetrization of $n$-operads and compactification of real configuration spaces, Adv. Math. 211 (2007), 684-725.

[3] Batanin M. A., The Eckmann-Hilton argument and higher operads, Adv. Math. 217 (2008), 334-385.

[4] Batanin M. A., Berger C., The lattice path operad and Hochschild cochains, Contemp. Math. 504 (2009), 23-52.

[5] Batanin M.A., Berger C., Homotopy theory of algebras over polynomial monads, arXiv:1305.0086.

[6] Berger C., Moerdijk I., Axiomatic homotopy theory for operads, Comment. Math. Helv. 78 (2003), 805-831.

[7] Boavida de Brito P., Weiss M., Spaces of smooth embeddings and configuration categories. J. Topol. 11 (2018), no. 1, 65-143. 
[8] Cisinski D.-C., Les préfaisceaux comme modèles des types d'homotopie, Astérisque, Soc. Math. France, 308, 2006.

[9] Ducoulombier J., Deloopings derived mapping spaces of bimodules over an operad, preprint, arXiv:1704.07062

[10] Ducoulombier J., Turchin V., Delooping manifold calculus tower on a closed disc, preprint, arXiv:1708.02203.

[11] Dwyer W., Hess K., Long knots and maps between operads, Geometry \& Topology 16 (2012), 919-955

[12] Fiedorowicz Z., Classifying Spaces of Topological Monoids and Categories, American Journal of Math., Vol.106, No. 2 (1984), 301-350.

[13] Gambino N., Kock J., Polynomial functors and polynomial monads, Math. Proc. Cambridge Philos. Soc. 154 (2013), no. 1, 153-192.

[14] Grothendieck A., Pursuing stacks, manuscript (1983).

[15] Hirschhorn P., Model Categories and their Localizations, Math. Surveys Monogr., vol. 99, Amer. Math. Soc., Providence, RI, 2003.

[16] Kock J., Joyal A., Batanin M., Mascari J.-F., Polynomial functors and opetopes, Adv. Math. 224 (2010), 2690-2737.

[17] Malsiniotis G., La théorie de l'homotopie de Grothendieck, Astérisque, 301, (2005) 1-140.

[18] Markl M., Operads and PROP's, in Elsevier, Handbook of Algebra vol. 5 (2008), 87-140.

[19] Quillen D.G., Higher algebraic K-theory, Lecture Notes in Math., vol. 341, Springer-Verlag,1973,p.85-147.

[20] Sinha Dev P., Operads and knot spaces, Journal of the American Mathematical Society 14 (2006), 461-486.

[21] Thomason R., Homotopy colimits in the category of small categories, Math. Proc. Camb. Phil. Soc., , 85, (1979), 91-109.

[22] Thomason R., Cat as a closed model category, Cahiers Topologie Géom. Diff., XXI-3, (1980), 305-324.

[23] Turchin V., Delooping totalization of a multiplicative operad, Journal of Homotopy and Related Structures 9 (2014), 349-418.

[24] Weber M., Internal algebra classifiers as codescent objects of crossed internal categories, Theory and Applications of Categories, 30:1713-1792, 2015.

[25] White D., Yau D., Bousfield localization and algebras over colored operads, arXiv:1503.06720 